\title{
Generation and Application of Nested Entanglement in Matryoshka Quantum Resource-States
}

\author{
Mrittunjoy Guha Majumdar* \\ University of Cambridge
}

(Dated: July 8, 2020)

\begin{abstract}
Multipartite entanglement is a resource for application in disparate protocols, of computing, communication and cryptography. Nested entanglement provides resource-states for quantum information processing. In this paper, Matryoshka quantum resource-states, which contain nested entanglement patterns, has been studied. A novel scheme for the generation of such quantum states has been proposed using an anisotropic XY spin-spin interaction-based model. The application of the Matryoshka GHZ-Bell states for n-qubit teleportation is reviewed and an extension to more general Matryoshka ExhS-Bell states is posited. An example of Matryoshka ExhS-Bell states is given in the form of the genuinely entangled seven-qubit Xin-Wei Zha state. Generation, characterisation and application of this seven-qubit resource state in theoretical schemes for quantum teleportation of arbitrary one, two and three qubits states, bidirectional teleportation of arbitrary two qubit states and probabilistic circular controlled teleportation are presented.
\end{abstract}

Keywords: Quantum Computation, Multipartite Entanglement, Quantum State Sharing

Introduction. Quantum Entanglement, along with other general non-local quantum correlations, has sparked a revolution in Physics that has led to the emergence of Quantum Information (processing) [1-10]. It has been used in applications such as teleportation and super-dense coding [11-13]. Bennet et al first proposed a scheme for quantum teleportation, wherein a genuinely entangled Bell state was used to transmit an arbitrary single qubit [14]. Many different kinds of entangled quantum states have been used to teleport arbitrary quantum states since then, including $\mathrm{W}$ states $[15,16]$, Bell states [17, 18], GHZ states [19, 20] and multiqubit states [21-23]. There have been hop-by-hop and multihop quantum teleportation schemes proposed since then, as well as schemes to teleport GHZ-like states using two types of four-qubit states [24, 25]. Teleportation has been proposed in two-copy quantum teleportation scheme [26], using cluster states [27], in higher dimensions [28] and also shown to be possible over atmosphere channels [29]. More recently, various derivatives of the standard teleportation scheme have been proposed, including those used for controlled teleportation [30,31], bidirectional teleportation [20,32-35], quantum secret sharing [36-38], quantum operation sharing [39, 40] and arbitrated quantum teleportation [41, 42]. What has also been of interest has been controlled teleportation of an entangled state in a noisy environment [42, 43].

This broad area of research around entanglement and teleportation in noisy environments is related to another important area of work that centres around decoherence, decoherence-free subspaces and Markovian as well as Non-Markovian quantum noises [44-52].

\footnotetext{
*mrittunjoy.guhamajumdar@cantab.net

Most of the work for this paper was done under affiliation to the University of Cambridge (2014-2019)
}

Entanglement has also been key in quantum communication protocols [53-55], with Quantum Repeaters and Entanglement Purification being the subject of interest lately [56-58]. For information transfer using entanglement between parties, one needs an established entangled channel-state and means of classical communication. For a large number of parties, multipartite entanglement and entangled multiqubit states play the preeminent role, with states varying from GHZ- and Wstates to clusters states [59,60]. Teleportation of an arbitrary single qubit state using a channel comprising of an EPR pair was first demonstrated by Bennett et al [14]. Lately, W-GHZ composite states have been used for teleportation, remote state sharing as well as superdense coding of arbitrary quantum states [61]. These composite (Matryoshka) quantum states contain nested entanglement, which can be used for applications in quantum information processing. In this paper, the generation, characterisation and application of Matryoshka GHZ-Bell and GHZ-ExhS quantum resource-states will be explored.

Generation of Matryoshka GHZ-Bell States. The primary resource state used as a resource in this study is a GHZ-Bell state. The concept of Matryoshka states was first given by Di Franco et al [62], with the name 'Matryoshka' coming from the Russian word for 'nesting doll'. In such states, we have a nested entanglement pattern. In this paper, we will be looking at the Matryoshka generation of the GHZ-Bell states over arbitrary number of qubits. We consider $N$ spin- $\frac{1}{2}$ particles, with each spin coupled to its nearest neighbors by the XY Hamiltonian

$$
H=\sum_{i=1}^{N-1}\left(J_{X, i} \hat{X}_{i} \hat{X}_{i+1}+J_{Y, i} \hat{Y}_{i} \hat{Y}_{i+1}\right)
$$

where $J_{\sigma, i}$ is the pairwise coupling constant with $\sigma=$ $\hat{X}, \hat{Y}, \hat{Z}$ being the Pauli operators. For the purposes of 
this paper, we take $N$ to be odd. Franco et al [62] showed that it is sufficient to state that the information flux between the $\hat{X}(\hat{Y})$ operators of the first and last qubits in the spin-chain depends on an alternating set of coupling strengths. For example, the information flux from $\hat{X}_{1}$ to $\hat{X}_{N}$ depends only on the set $\left\{J_{Y, 1}, J_{X, 2}, \ldots, J_{Y, N-1}\right\}$ and is independent of any other coupling rate in the spinchain. Christandl et al $[63,64]$ showed that after a time $t^{*}=\pi / \lambda$ with $\lambda$ being a scaling constant (as mentioned in the definition of the case of a perfect state transfer in a linear spin-chain given by weighted coupling strengths: $\left.J_{\sigma, i}=\lambda \sqrt{i(N-i)}\right)$, the state of the first qubit in the spin-chain can be perfectly transferred to the last qubit. We see that by preparing the initial state of this spinchain in an completely separable eigenstate of the tensorial product of $Z_{i}$ operators, say $|\Psi(0)\rangle=|000 \ldots 0\rangle_{12 \ldots N}$, we obtain an information flux towards symmetric twosite spin operators, and a final state of the form [62]

$$
\begin{aligned}
& \left|\psi_{0}\right\rangle=|0\rangle_{c} \otimes_{i=0}^{M}\left|\psi_{+}\right\rangle_{2 i+1, N-2 i} \otimes_{i=1}^{M}\left|\psi_{-}\right\rangle_{2 i, N-2 i+1} \\
& \left|\psi_{1}\right\rangle=|1\rangle_{c} \otimes_{i=0}^{M}\left|\psi_{-}\right\rangle_{2 i+1, N-2 i} \otimes_{i=1}^{M}\left|\psi_{+}\right\rangle_{2 i, N-2 i+1}
\end{aligned}
$$

where $c$ labels the central site of the spin-chain, $M=\frac{N-3}{4}$ and $\left|\psi_{ \pm}\right\rangle=\frac{1}{\sqrt{2}}(|00\rangle \pm|11\rangle)$. An illustration of the setup has been shown in Figure 1.

The critical step in the creation of the Matryoshka GHZ-Bell state is the evolution of the central and two neighbouring qubits to the GHZ state, without disturbing the rest of the spin-chain. For this, we need to switch off all the interactions except for those connecting the central qubit to the neighbouring ones. A point to note here is that had we started with $|\Psi(0)\rangle=|111 \ldots 1\rangle_{12 \ldots N}$, we would have obtained a final state of the form

$$
\begin{aligned}
& \left|\psi_{0}\right\rangle=|0\rangle_{c} \otimes_{i=0}^{M}\left|\psi_{-}\right\rangle_{2 i+1, N-2 i} \otimes_{i=1}^{M}\left|\psi_{+}\right\rangle_{2 i, N-2 i+1} \\
& \left|\psi_{1}\right\rangle=|1\rangle_{c} \otimes_{i=0}^{M}\left|\psi_{+}\right\rangle_{2 i+1, N-2 i} \otimes_{i=1}^{M}\left|\psi_{-}\right\rangle_{2 i, N-2 i+1}
\end{aligned}
$$

We use this principle and the idea that after evolution over time $t^{*}$, the states in equations (2) and (3) transform back to $|000 \ldots 000\rangle_{12 \ldots N}$ and states in equations (4) and (5) transform back to $|111 \ldots 11\rangle_{12 \ldots N}$. We can utilise this concept, by taking the state in equation (2) and evolving it, for the truncated subsystem comprising of the central qubit and the adjoining qubits. A point to note here is that due to only coupling that connects to the central qubits, the coupling strength $\left(J_{\sigma, i}^{\prime}=\lambda^{\prime} \sqrt{i(3-i)}\right)$ and time of evolution $\left(t^{\prime \prime}=\pi / \lambda^{\prime}\right)$ vary accordingly. Before carrying out this evolution, we perform a Hadamard operation on the central qubit to give

$$
\begin{array}{r}
\left|\psi_{0}\right\rangle=\frac{1}{\sqrt{2}}\left(|0\rangle_{c}+|1\rangle_{c}\right) \otimes_{i=0}^{M}\left|\psi_{+}\right\rangle_{2 i+1, N-2 i} \\
\otimes_{i=1}^{M}\left|\psi_{-}\right\rangle_{2 i, N-2 i+1}
\end{array}
$$

We now perform the truncated subsystem time-evolution with the parameters $\left(J^{\prime}, t^{\prime \prime}\right)$ to give us the state

$$
\begin{array}{r}
\left|\psi_{0}\right\rangle=\frac{1}{\sqrt{2}}(|000\rangle+|111\rangle)_{c-1, c, c+1} \otimes_{i=0}^{M-1}\left|\psi_{+}\right\rangle_{2 i+1, N-2 i} \\
\otimes_{i=1}^{M}\left|\psi_{-}\right\rangle_{2 i, N-2 i+1}
\end{array}
$$

Therefore, we can obtain a Matryoshka GHZ-Bell state using nearest spin-spin interactions in a spin-chain.

Teleportation of arbitrary n-qubit quantum states using Matryoshka GHZ-Bell States. The teleportation of an arbitrary n-qubit state can be performed using Matryoshka GHZ-Bell States. Saha et al proposed a scheme for teleportation of a multiqubit state using the following resource state [65]:

$$
\begin{array}{r}
|\psi\rangle=\left[\frac{1}{\sqrt{2}}\left(\left|0_{A} 0_{A} 0_{B}\right\rangle+\left|1_{A} 1_{A} 1_{B}\right\rangle\right)\right]\left[\frac{1}{\sqrt{2}}\left(\left|0_{A} 0_{B}\right\rangle+\left|1_{A} 1_{B}\right\rangle\right)\right] \\
\ldots\left[\frac{1}{\sqrt{2}}\left(\left|0_{A} 0_{B}\right\rangle+\left|1_{A} 1_{B}\right\rangle\right)\right]
\end{array}
$$

To achieve the teleportation of an $n$-qubit state, we start with a $2 n+1$ qubit state of the form given in equation (8), with Alice having $n+1$ qubits and Bob having $n$ qubits. Let us say the arbitrary $n$-qubit state Alice wants to teleport to Bob is: $\sum_{i=0}^{2^{n}-1} \alpha_{i}\left|a_{i}\right\rangle$, where $a_{i}$ denotes the binary representation of $i$. The combined state $\left|\psi_{c}\right\rangle$ can then be written in terms of subsystems possessed by Alice and Bob,

$$
\left|\psi_{c}\right\rangle=\sum_{i=0}^{2^{2 n}-1}\left|\omega_{i}\right\rangle_{A}\left|\eta_{i}\right\rangle_{B}
$$

where $\left|\omega_{i}\right\rangle_{A} \forall i \in\left[0,2^{2 n}-1\right]$ constitute a mutually orthogonal basis. It is seen that for even $n$, $\left|\omega_{0}\right\rangle_{1,4,3,2,7, \ldots, 2 n, 2 n-3,2 n-2,2 n+1}=\left|G H Z_{+}\right\rangle\left|\psi_{+}\right\rangle \ldots\left|\psi_{+}\right\rangle$ and for odd $n, \quad\left|\omega_{0}\right\rangle_{1,2,5,4,3, \ldots, 2 n, 2 n-3,2 n-2,2 n+1}=$ $\left|G H Z_{+}\right\rangle\left|\psi_{+}\right\rangle \ldots\left|\psi_{+}\right\rangle$, where $\left|G H Z_{+}\right\rangle=\frac{1}{\sqrt{2}}(|000\rangle+|111\rangle)$ and $\left|\psi_{+}\right\rangle=\frac{1}{\sqrt{2}}(|00\rangle+|11\rangle)[65]$. We can obtain the other $\left|\omega_{i}\right\rangle_{A}$ from this

$\left|\omega_{i}\right\rangle_{1,2,3, \ldots, 2 n, 2 n+1}=\otimes_{k=1}^{n}\left(Z_{k}\right)^{b_{k}}\left(X_{k}\right)^{b_{k+n}}\left|\omega_{0}\right\rangle_{1,2,3, \ldots, 2 n, 2 n+1}$

where $i$ is the decimal representation of the string $b_{2 n} \ldots \ldots b_{2} b_{1}$ with a general $b_{k}$ being 0 or 1 . After measurement is done using these orthogonal states, Alice's state evolves into one of the states $\left|\omega_{i}\right\rangle^{\prime}$. If her state is $\left|\omega_{i}\right\rangle$ then Bob must apply $\otimes_{k=1}^{n}\left(Z_{k}\right)^{b_{k}}\left(X_{k}\right)^{b_{k+n}}$ on his n-qubit system to obtain the unknown state, where $i$ is the decimal representation of the string $b_{2 n} \ldots \ldots b_{2} b_{1}$ with a general $b_{k}$ being 0 or 1 .

Extension of Matryoshka formalism to ExhS-Bell States. We can extend the idea of nested entanglement (Matryoshka formalism) to the case of ExhS-Bell States, which comprise of the exhaustive set over the basis for 
the qubit states in the ExhS subsystem alongwith the maximally entangled Bell-states for the remainder subsystem. Such states are found to be less entangled in the three-qubit subsystem but have greater nested entangled resources. An example of Matryoshka ExhS-Bell States are the Xin-Wei Zha (XZW) State. Xin-Wei Zha et al [66] discovered a genuinely entangled seven-qubit state through a numerical optimization process, following the path taken by Brown et al [67] and Borras et al [68] to find genuinely entangled five-qubit and six-qubit states:

$$
\begin{aligned}
& \left|\psi_{7}\right\rangle=\frac{1}{2 \sqrt{2}}\left(|000\rangle_{135}\left|\psi_{+}\right\rangle_{24}\left|\psi_{+}\right\rangle_{67}+|001\rangle_{135}\left|\phi_{-}\right\rangle_{24}\left|\phi_{+}\right\rangle_{67}\right. \\
& \quad+|010\rangle_{135}\left|\psi_{-}\right\rangle_{24}\left|\phi_{-}\right\rangle_{67}+|011\rangle_{135}\left|\phi_{+}\right\rangle_{24}\left|\psi_{-}\right\rangle_{67} \\
& \quad+|100\rangle_{135}\left|\phi_{+}\right\rangle_{24}\left|\phi_{+}\right\rangle_{67}+|101\rangle_{135}\left|\psi_{-}\right\rangle_{24}\left|\psi_{+}\right\rangle_{67} \\
& \left.+|110\rangle_{135}\left|\phi_{-}\right\rangle_{24}\left|\psi_{-}\right\rangle_{67}+|111\rangle_{135}\left|\psi_{+}\right\rangle_{24}\left|\phi_{-}\right\rangle_{67}\right)
\end{aligned}
$$

It is seen that this state can be used for teleportation of arbitrary single, double and triple qubit states. The 3-2-2 structure of the resource-state, given in equation (11), helps us in devising a quantum circuit to generate the state, as shown in Figure 2 and realised on IBM Quantum Experience, giving us the state

$$
\begin{aligned}
|\psi\rangle & =\frac{1}{2 \sqrt{2}}\left(|000\rangle_{135}\left|\psi_{+}\right\rangle_{24}\left|\psi_{+}\right\rangle_{67}+|001\rangle_{135}\left|\phi_{-}\right\rangle_{24}\left|\phi_{+}\right\rangle_{67}\right. \\
& +|010\rangle_{135}\left|\psi_{-}\right\rangle_{24}\left|\phi_{-}\right\rangle_{67}+|011\rangle_{135}\left|\phi_{+}\right\rangle_{24}\left|\psi_{-}\right\rangle_{67} \\
& +|100\rangle_{135}\left|\phi_{+}\right\rangle_{24}\left|\phi_{+}\right\rangle_{67}+|101\rangle_{135}\left|\psi_{-}\right\rangle_{24}\left|\psi_{+}\right\rangle_{67} \\
+ & \left.|110\rangle_{135}\left|\phi_{-}\right\rangle_{24}\left|\psi_{-}\right\rangle_{67}+|111\rangle_{135}\left|\psi_{+}\right\rangle_{24}\left|\phi_{-}\right\rangle_{67}\right)
\end{aligned}
$$

To obtain the resource-state, we apply a unitary operator on qubits 1,3 and 5: $U=I_{4 \times 4} \oplus\left(\sigma_{z} \otimes \sigma_{z}\right)$. This state has marginal density matrices for subsystems over one or two qubits that are completely mixed, with

$$
\begin{gathered}
\pi_{i j}=\operatorname{Tr}_{i j} \rho_{i j}^{2}=\frac{1}{4} \forall i, j \in\{1,2,3,4,5,6,7\}, i<j \\
\pi_{i}=\operatorname{Tr}_{i} \rho_{i}^{2}=\frac{1}{2} \forall i \in\{1,2,3,4,5,6,7\}
\end{gathered}
$$

For three-qubit subsystems, some of the partitions have mixed marginal density matrices:

$$
\begin{gathered}
\pi_{i j k}=T r_{i j k} \rho_{i j k}^{2}=\frac{1}{8} \forall i, j \in\{1,2,3,4,5,6,7\} \\
i<j<k \wedge(i j k) \neq(127),(367),(457) \\
\pi_{127}=\pi_{367}=\pi_{457}=\frac{1}{4}
\end{gathered}
$$

Linear, Bidirectional and Circular Quantum Teleportation using the $X Z W$ Resource State. The seven-qubit genuinely entangled resource state $\left|\Gamma_{7}\right\rangle$ can be used for a number of applications, such as the teleportation of arbitrary one, two and three qubit states. To begin with, an arbitrary single qubit state can be teleported using the resource state $\left|\Gamma_{7}\right\rangle$ will be considered. In this case Alice possesses qubits 1, 2, 3, 4, 5, 6 and the 7th particle belongs to Bob. Alice wants to transport an arbitrary state $\left|\psi^{(1)}\right\rangle=\alpha|0\rangle+\beta|1\rangle$ to Bob. The combined state of the system is $\left|\Gamma_{7}^{(1)}\right\rangle=\left|\psi^{(1)}\right\rangle \otimes\left|\Gamma_{7}\right\rangle$. Alice measures the seven qubits in her possession via the seven qubit orthonormal states: $\left|\xi^{ \pm}\right\rangle=|0000\rangle\left|\Psi_{G H Z}^{0}\right\rangle-$ $|0001\rangle\left|\Psi_{G H Z}^{3}\right\rangle+|0010\rangle\left|\Psi_{G H Z}^{7}\right\rangle+|0011\rangle\left|\Psi_{G H Z}^{4}\right\rangle-$ $|0100\rangle\left|\Psi_{G H Z}^{5}\right\rangle-|0101\rangle\left|\Psi_{G H Z}^{6}\right\rangle+|0110\rangle\left|\Psi_{G H Z}^{2}\right\rangle+$ $|0111\rangle\left|\Psi_{G H Z}^{1}\right\rangle \pm\left(|1000\rangle\left|\Psi_{G H Z}^{2}\right\rangle-|1001\rangle\left|\Psi_{G H Z}^{1}\right\rangle-\right.$ $|1010\rangle\left|\Psi_{G H Z}^{5}\right\rangle-|1011\rangle\left|\Psi_{G H Z}^{6}\right\rangle+|1100\rangle\left|\Psi_{G H Z}^{7}\right\rangle+$ $\left.|1101\rangle\left|\Psi_{G H Z}^{4}\right\rangle+|1110\rangle\left|\Psi_{G H Z}^{0}\right\rangle-|1111\rangle\left|\Psi_{G H Z}^{3}\right\rangle\right)$ and $\left|\nu^{ \pm}\right\rangle=|1000\rangle\left|\Psi_{G H Z}^{0}\right\rangle-|1001\rangle\left|\Psi_{G H Z}^{3}\right\rangle+|1010\rangle\left|\Psi_{G H Z}^{7}\right\rangle+$ $|1011\rangle\left|\Psi_{G H Z}^{4}\right\rangle-|1100\rangle\left|\Psi_{G H Z}^{5}\right\rangle-|1101\rangle\left|\Psi_{G H Z}^{6}\right\rangle+$ $|1110\rangle\left|\Psi_{G H Z}^{2}\right\rangle+|1111\rangle\left|\Psi_{G H Z}^{1}\right\rangle \pm\left(|0000\rangle\left|\Psi_{G H Z}^{2}\right\rangle-\right.$ $|0001\rangle\left|\Psi_{G H Z}^{1}\right\rangle-|0010\rangle\left|\Psi_{G H Z}^{5}\right\rangle-|0011\rangle\left|\Psi_{G H Z}^{6}\right\rangle+$ $|0100\rangle\left|\Psi_{G H Z}^{7}\right\rangle+|0101\rangle\left|\Psi_{G H Z}^{4}\right\rangle+|0110\rangle\left|\Psi_{G H Z}^{0}\right\rangle-$ $\left.|0111\rangle\left|\Psi_{G H Z}^{3}\right\rangle\right)$, where $\left|\Psi_{G H Z}^{0,1}\right\rangle=\frac{1}{\sqrt{2}}[|000\rangle \pm|111\rangle]$, $\left|\Psi_{G H Z}^{2,3}\right\rangle=\frac{1}{\sqrt{2}}[|001\rangle \pm|110\rangle],\left|\Psi_{G H Z}^{4,5}\right\rangle=\frac{1}{\sqrt{2}}[|010\rangle \pm|101\rangle]$ and $\left|\Psi_{G H Z}^{6,7}\right\rangle=\frac{1}{\sqrt{2}}[|100\rangle \pm|011\rangle]$.

Alice then conveys the outcome of the measurement results to Bob via two classical bits. Bob then applies a suitable unitary operation from the set $I, \sigma_{x}, i \sigma_{y}, \sigma_{z}$ to recover the original state, sent by Alice. In this way, one can teleport an arbitrary single-qubit state using the state $\left|\Gamma_{7}\right\rangle$. Similarly, an arbitrary two qubit quantum state can be teleported using the resource-state. In this case Alice possesses qubits 1, 2, 3, 4 and 5, and the 6th and 7th particles belong to Bob. Alice wants to transport an arbitrary state $\left|\psi^{(2)}\right\rangle=\alpha|00\rangle+\mu|10\rangle+\gamma|01\rangle+\beta|11\rangle$ to Bob. The combined state of the system is $\left|\Gamma_{7}^{(2)}\right\rangle=\left|\psi^{(2)}\right\rangle \otimes\left|\Gamma_{7}\right\rangle$,

$$
\begin{aligned}
& \left|\Gamma_{7}^{(2)}\right\rangle=\alpha\left(A_{00}|00\rangle+A_{01}|01\rangle+A_{10}|10\rangle+A_{11}|11\rangle\right) \\
& \quad+\mu\left(B_{00}|00\rangle+B_{01}|01\rangle+B_{10}|10\rangle+B_{11}|11\rangle\right) \\
& \quad+\gamma\left(C_{00}|00\rangle+C_{01}|01\rangle+C_{10}|10\rangle+C_{11}|11\rangle\right) \\
& +\beta\left(D_{00}|00\rangle+D_{01}|01\rangle+D_{10}|10\rangle+D_{11}|11\rangle\right)
\end{aligned}
$$

where $A_{00}=|0000\rangle\left|\Psi_{G H Z}^{0}\right\rangle+|0001\rangle\left|\Psi_{G H Z}^{4}\right\rangle-$ $|0010\rangle\left|\Psi_{G H Z}^{5}\right\rangle-|0011\rangle\left|\Psi_{G H Z}^{7}\right\rangle, A_{11}=|0000\rangle\left|\Psi_{G H Z}^{1}\right\rangle+$ $|0001\rangle\left|\Psi_{G H Z}^{5}\right\rangle-|0010\rangle\left|\Psi_{G H Z}^{2}\right\rangle-|0011\rangle\left|\Psi_{G H Z}^{7}\right\rangle$, $A_{01}=|0000\rangle\left|\Psi_{G H Z}^{6}\right\rangle-|0001\rangle\left|\Psi_{G H Z}^{2}\right\rangle+|0010\rangle\left|\Psi_{G H Z}^{4}\right\rangle+$ $|0011\rangle\left|\Psi_{G H Z}^{0}\right\rangle, A_{10}=-|0000\rangle\left|\Psi_{G H Z}^{7}\right\rangle-|0001\rangle\left|\Psi_{G H Z}^{3}\right\rangle+$ $|0010\rangle\left|\Psi_{G H Z}^{5}\right\rangle+|0011\rangle\left|\Psi_{G H Z}^{1}\right\rangle, B_{00}=|1000\rangle\left|\Psi_{G H Z}^{0}\right\rangle+$ $|1001\rangle\left|\Psi_{G H Z}^{4}\right\rangle-|1010\rangle\left|\Psi_{G H Z}^{2}\right\rangle+|1011\rangle\left|\Psi_{G H Z}^{6}\right\rangle$, $B_{11}=|1000\rangle\left|\Psi_{G H Z}^{0}\right\rangle+|1001\rangle\left|\Psi_{G H Z}^{5}\right\rangle-|1010\rangle\left|\Psi_{G H Z}^{3}\right\rangle-$ $|1011\rangle\left|\Psi_{G H Z}^{7}\right\rangle, B_{01}=|1000\rangle\left|\Psi_{G H Z}^{6}\right\rangle-|1001\rangle\left|\Psi_{G H Z}^{2}\right\rangle+$ $|1010\rangle\left|\Psi_{G H Z}^{4}\right\rangle+|1011\rangle\left|\Psi_{G H Z}^{0}\right\rangle, B_{10}=-|1000\rangle\left|\Psi_{G H Z}^{7}\right\rangle-$ $|1001\rangle\left|\Psi_{G H Z}^{3}\right\rangle+|1010\rangle\left|\Psi_{G H Z}^{5}\right\rangle+|1011\rangle\left|\Psi_{G H Z}^{1}\right\rangle$, $C_{00}=|0100\rangle\left|\Psi_{G H Z}^{0}\right\rangle+|0101\rangle\left|\Psi_{G H Z}^{4}\right\rangle-|0110\rangle\left|\Psi_{G H Z}^{3}\right\rangle+$ $|0111\rangle\left|\Psi_{G H Z}^{6}\right\rangle, C_{11}=|0100\rangle\left|\Psi_{G H Z}^{1}\right\rangle+|0101\rangle\left|\Psi_{G H Z}^{5}\right\rangle-$ $|0110\rangle\left|\Psi_{G H Z}^{3}\right\rangle-|0111\rangle\left|\Psi_{G H Z}^{7}\right\rangle, C_{01}=|0100\rangle\left|\Psi_{G H Z}^{6}\right\rangle-$ $|0101\rangle\left|\Psi_{G H Z}^{2}\right\rangle-|0110\rangle\left|\Psi_{G H Z}^{4}\right\rangle+|0111\rangle\left|\Psi_{G H Z}^{0}\right\rangle$, $C_{10}=|0100\rangle\left|\Psi_{G H Z}^{7}\right\rangle-|0101\rangle\left|\Psi_{G H Z}^{3}\right\rangle+|0110\rangle\left|\Psi_{G H Z}^{5}\right\rangle+$ $|0111\rangle\left|\Psi_{G H Z}^{1}\right\rangle, D_{00}=|1100\rangle\left|\Psi_{G H Z}^{0}\right\rangle+|1101\rangle\left|\Psi_{G H Z}^{4}\right\rangle-$ 
$|1110\rangle\left|\Psi_{G H Z}^{2}\right\rangle+|1111\rangle\left|\Psi_{G H Z}^{6}\right\rangle, D_{11}=|1100\rangle\left|\Psi_{G H Z}^{1}\right\rangle+$ $|1101\rangle\left|\Psi_{G H Z}^{5}\right\rangle-|1110\rangle\left|\Psi_{G H Z}^{3}\right\rangle-|1111\rangle\left|\Psi_{G H Z}^{7}\right\rangle$, $D_{01}=|1100\rangle\left|\Psi_{G H Z}^{6}\right\rangle-|1101\rangle\left|\Psi_{G H Z}^{2}\right\rangle+|1110\rangle\left|\Psi_{G H Z}^{4}\right\rangle+$ $|1111\rangle\left|\Psi_{G H Z}^{0}\right\rangle, D_{10}=|1100\rangle\left|\Psi_{G H Z}^{6}\right\rangle-|1101\rangle\left|\Psi_{G H Z}^{3}\right\rangle+$ $|1110\rangle\left|\Psi_{G H Z}^{5}\right\rangle+|1111\rangle\left|\Psi_{G H Z}^{1}\right\rangle$, where $\left|\Psi_{G H Z}^{0,1}\right\rangle=$ $\frac{1}{\sqrt{2}}[|000\rangle \pm|111\rangle],\left|\Psi_{G H Z}^{2,3}\right\rangle=\frac{1}{\sqrt{2}}[|001\rangle \pm|110\rangle],\left|\Psi_{G H Z}^{4,5}\right\rangle=$ $\frac{1}{\sqrt{2}}[|010\rangle \pm|101\rangle]$ and $\left|\Psi_{G H Z}^{6,7}\right\rangle=\frac{1}{\sqrt{2}}[|100\rangle \pm|011\rangle]$. Now, Bob can carry out a combination of unitary operations, according to the given table, to obtain the original state teleported by Alice.

\begin{tabular}{|c|c|}
\hline State Obtained by Alice & Unitary Operation by Bob \\
\hline$A_{01}+B_{11}+C_{00}+D_{01}$ & $I \otimes \sigma_{x}$ \\
\hline$A_{01}+B_{11}-C_{00}-D_{01}$ & $\sigma_{z} \otimes \sigma_{x}$ \\
\hline$A_{01}-B_{11}+C_{00}-D_{01}$ & $I \otimes i \sigma_{y}$ \\
\hline$A_{01}-B_{11}-C_{00}+D_{01}$ & $\sigma_{z} \otimes i \sigma_{y}$ \\
\hline$A_{11}+B_{01}+C_{10}+D_{00}$ & $\sigma_{x} \otimes \sigma_{x}$ \\
\hline$A_{11}-B_{01}+C_{10}-D_{00}$ & $\sigma_{x} \otimes i \sigma_{y}$ \\
\hline$A_{11}+B_{01}-C_{10}-D_{00}$ & $i \sigma_{y} \otimes \sigma_{x}$ \\
\hline$A_{11}-B_{01}-C_{10}+D_{00}$ & $i \sigma_{y} \otimes i \sigma_{y}$ \\
\hline$A_{00}+B_{10}+C_{01}+D_{11}$ & $I \otimes I$ \\
\hline$A_{00}-B_{10}+C_{01}-D_{11}$ & $I \otimes \sigma_{z}$ \\
\hline$A_{00}+B_{10}-C_{01}-D_{11}$ & $\sigma_{z} \otimes I$ \\
\hline$A_{00}-B_{10}-C_{01}+D_{11}$ & $\sigma_{z} \otimes \sigma_{z}$ \\
\hline$A_{10}+B_{11}+C_{00}+D_{01}$ & $\sigma_{x} \otimes I$ \\
\hline$A_{10}-B_{11}+C_{00}-D_{01}$ & $\sigma_{x} \otimes \sigma_{z}$ \\
\hline$A_{10}+B_{11}-C_{00}-D_{01}$ & $i \sigma_{y} \otimes I$ \\
\hline$A_{10}-B_{11}-C_{00}+D_{01}$ & $i \sigma_{y} \otimes \sigma_{z}$ \\
\hline
\end{tabular}

The resource-state can also be used for bidirectional quantum teleportation. Bidirectional Controlled Quantum Teleportation (BCQT) protocols have been proposed for multi-qubit resource states, such as fivequbit [69], six-qubit [70, 71], seven-qubit [34, 72, 73] and eight-qubit states [74]. Bidirectional Controlled Quantum Teleportation can teleport arbitrary states between two users under the supervision of a third party. Zha et al proposed the first scheme for BCQT of single qubit states using a maximally entangled seve-qubit quantum state [34]. There have been schemes proposed for BCQT that utilise states with the same number of qubits as the quantum channel being used, and thereby realise bidirectional teleportation of arbitrary singleand two-qubit states under the controller Charlie [72, 73].

Let us say Alice and Bob would like to teleport two-qubit states to each other by utilizing the sevenqubit genuinely entangled resource state. We assume the form of the two-qubit states to be

$$
\begin{array}{r}
|\phi\rangle_{A_{1} A_{2}}=\alpha_{0}|00\rangle+\alpha_{1}|01\rangle+\alpha_{2}|10\rangle+\alpha_{3}|11\rangle \\
|\phi\rangle_{B_{1} B_{2}}=\beta_{0}|00\rangle+\beta_{1}|01\rangle+\beta_{2}|10\rangle+\beta_{3}|11\rangle
\end{array}
$$

For the resource-state, let Alice have the qubits 1,4 and 7, while Bob has the qubits 2, 3 and 6 and Charlie has the qubit 5.

The steps for the scheme are as follows:
- Alice measures qubit 7 of the resource state and $A_{1}$ in the bell basis.

- Bob measures qubit 2 of the resource state and $B_{1}$ in the bell basis.

- Charlie, Alice and Bob measure their qubits in the Z-basis.

- Alice and Bob measure their qubits $A_{2}$ and $B_{2}$ in the X-basis.

- We apply unitary transformations to the composite state to now get Alice's initial arbitrary state in Bob's terminal and Bob's initial arbitrary state in Alice's terminal.

Due to the special 3-2-2 form of the resource-state, we can also use entanglement swapping to perform bidirectional teleportation [75].

The seven-qubit resource state can be used for the perfect linear teleportation of an arbitrary three qubit state. In this case, Alice possesses qubits 1, 2, 3, 4 and 5, and the 6 th and 7 th particles belong to Bob. Alice wants to transport an arbitrary state $\left|\psi^{(3)}\right\rangle=a|000\rangle+b|001\rangle+$ $c|010\rangle+d|011\rangle+e|100\rangle+f|101\rangle+g|110\rangle+h|111\rangle$ to Bob. Using the decomposition given in Supplementary Material, the states possessed by, and the unitary transforms to be performed by, Bob have been recorded, to accomplish the teleportation of an arbitrary three-qubit state. A point to note here is that we get the GHZ state for $a=h=\frac{1}{\sqrt{2}}, b=c=d=e=f=g=0$ and the $\mathrm{W}$ state for $b=c=e=\frac{1}{\sqrt{3}}, a=d=f=g=h=0$.

Not only is the seven-qubit resource state useful for linear and bidirectional teleportation but can also facilitate the probabilistic teleportation of an arbitrary single-qubit states in a circular manner between three network-nodes (users). Let us say I have Alice, Bob and Charlie in the system, with the first qubit used as a control qubit, qubits 1 and 4 given to Alice, qubits 2 and 6 given to Bob and qubits 3 and 7 given to Charlie. Let us say the arbitrary states are $\left|\psi_{A}\right\rangle=\alpha_{A}\left|0_{A}\right\rangle+\beta_{A}\left|1_{A}\right\rangle,\left|\psi_{B}\right\rangle=\alpha_{B}\left|0_{B}\right\rangle+\beta_{B}\left|1_{B}\right\rangle$ and $\left|\psi_{C}\right\rangle=\alpha_{C}\left|0_{C}\right\rangle+\beta_{C}\left|1_{C}\right\rangle$. Then, the composite state is given by $\left|\psi_{A}\right\rangle \otimes\left|\psi_{B}\right\rangle \otimes\left|\psi_{C}\right\rangle \otimes\left|\Gamma_{7}\right\rangle_{T A_{1} B_{1} C_{1} A_{1} B_{1} C_{1}}$, where $\left|\Gamma_{7}\right\rangle_{T}$ is the control qubit. We apply a CNOT gate using the qubits $\mathrm{A}, \mathrm{B}$ and $\mathrm{C}$ of the arbitrary states as the control-qubits and the first qubits of each user as the target-qubit. Let us for simplicity only consider the case where $\left|\Gamma_{7}\right\rangle_{T}=|0\rangle$. Let us now measure the first qubits of Alice, Bob and Charlie in the Z-basis. Let us say $\left|\Gamma_{7}\right\rangle_{A_{1} B_{1} C_{1}}=|010\rangle$, then we have the state $\left|\psi^{\prime \prime}\right\rangle=\frac{1}{4}\left((|001\rangle-|010\rangle) \alpha_{A} \alpha_{B} \alpha_{C}\left|0_{A} 0_{B} 0_{C}\right\rangle+\right.$

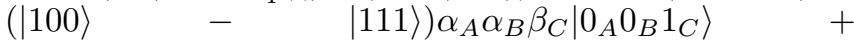
$(|000\rangle+|011\rangle) \alpha_{A} \beta_{B} \alpha_{C}\left|0_{A} 1_{B} 0_{C}\right\rangle+$ $(|101\rangle+|110\rangle) \alpha_{A} \beta_{B} \beta_{C}\left|0_{A} 1_{B} 1_{C}\right\rangle+(|100\rangle-$ $|111\rangle) \beta_{A} \alpha_{B} \alpha_{C}\left|1_{A} 0_{B} 0_{C}\right\rangle+(-|001\rangle+$ $|010\rangle) \beta_{A} \alpha_{B} \beta_{C}\left|1_{A} 0_{B} 1_{C}\right\rangle \quad+\quad(-|101\rangle \quad-$ 
$|110\rangle) \beta_{A} \beta_{B} \alpha_{C}\left|1_{A} 1_{B} 0_{C}\right\rangle+\quad+\quad(|000\rangle+$ $\left.|011\rangle) \beta_{A} \beta_{B} \beta_{C}\left|1_{A} 1_{B} 1_{C}\right\rangle\right)$. We can now measure the control qubits in the X-basis. So, let us say, I have $\left|Q_{A} Q_{B} Q_{C}\right\rangle=\left|+_{A}-{ }_{B}+{ }_{C}\right\rangle$, then we obtain the state $\left|C_{1}\right\rangle\left(\left|A_{1}\right\rangle\left(-\left|B_{1}\right\rangle+\chi\left|B_{3}\right\rangle-\chi^{-1}\left|B_{4}\right\rangle-\left|B_{2}\right\rangle\right)+\left|A_{2}\right\rangle\left(-\left|B_{1}\right\rangle+\right.\right.$ $\left.\left.\chi\left|B_{3}\right\rangle+\chi^{-1}\left|B_{4}\right\rangle+\left|B_{2}\right\rangle\right)\right)+\left|C_{2}\right\rangle\left(\left|A_{1}\right\rangle\left(-\left|B_{1}\right\rangle-\chi\left|B_{3}\right\rangle-\right.\right.$ $\left.\left.\chi^{-1}\left|B_{4}\right\rangle+\left|B_{2}\right\rangle\right)+\left|A_{2}\right\rangle\left(-\left|B_{1}\right\rangle-\chi\left|B_{3}\right\rangle+\chi^{-1}\left|B_{4}\right\rangle-\left|B_{2}\right\rangle\right)\right)+$ $\left|C_{3}\right\rangle\left(\left|A_{1}\right\rangle\left(\left|B_{4}\right\rangle-\chi\left|B_{2}\right\rangle-\chi^{-1}\left|B_{1}\right\rangle-\left|B_{3}\right\rangle\right)+\left|A_{2}\right\rangle\left(\left|B_{4}\right\rangle-\right.\right.$ $\left.\left.\chi\left|B_{2}\right\rangle+\chi^{-1}\left|B_{1}\right\rangle-\left|B_{3}\right\rangle\right)\right)+\left|C_{4}\right\rangle\left(\left|A_{1}\right\rangle\left(\left|B_{4}\right\rangle+\chi\left|B_{2}\right\rangle-\right.\right.$ $\left.\left.\chi^{-1}\left|B_{1}\right\rangle-\left|B_{3}\right\rangle\right)+\left|A_{2}\right\rangle\left(\left|B_{4}\right\rangle+\chi\left|B_{2}\right\rangle+\chi^{-1}\left|B_{1}\right\rangle+\left|B_{3}\right\rangle\right)\right)$, where $\left|C_{1}\right\rangle=\beta_{C}|0\rangle+\alpha_{C}|1\rangle,\left|C_{2}\right\rangle=\beta_{C}|0\rangle-\alpha_{C}|1\rangle$, $\left|C_{3}\right\rangle=\beta_{C}|1\rangle+\alpha_{C}|0\rangle, \quad\left|C_{4}\right\rangle=\beta_{C}|1\rangle-\alpha_{C}|0\rangle$, $\left|B_{1}\right\rangle=\alpha_{B}|1\rangle+\beta_{B}|0\rangle, \quad\left|B_{2}\right\rangle=\alpha_{B}|1\rangle-\beta_{B}|0\rangle$, $\left|B_{3}\right\rangle=\alpha_{B}|0\rangle+\beta_{B}|1\rangle,\left|B_{4}\right\rangle=\alpha_{B}|0\rangle-\beta_{B}|1\rangle, \chi=\frac{a_{2}}{a_{1}}$ with $a_{1}=\beta_{A}+\alpha_{A}, a_{2}=\alpha_{A}-\beta_{A},\left|A_{1}\right\rangle=a_{1}|0\rangle+a_{2}|1\rangle$, $\left|A_{1}\right\rangle=a_{1}|0\rangle-a_{2}|1\rangle$. Therefore I see that the users can obtain states derived from the original state of the users next to them (Alice $\rightarrow$ Bob $\rightarrow$ Charlie $\rightarrow$ Alice). However, as you can see, this can be done in a probabilistic manner with one of the users not quite obtaining the original state but rather a derivative-state based on the original.

Conclusion. In this paper, the generation and application of nested entanglement in Matryoshka resource-states for quantum information processing was studied. A novel scheme for the generation of such quantum states has been proposed using an anisotropic XY spin-spin interaction-based model. The application of the Matryoshka GHZ-Bell states for n-qubit teleportation is reviewed and an extension to more general Matryoshka ExhS-Bell states is posited. An example of Matryoshka ExhS-Bell states was given in the form of the genuinely entangled seven-qubit Xin-Wei Zha state. Generation, characterisation and application of this seven-qubit resource state in theoretical schemes for quantum teleportation of arbitrary one, two and three qubits states, bidirectional teleportation of arbitrary two qubit states and probabilistic circular controlled teleportation were presented. This work should lay the groundwork for other studies into the area of nested entanglement.

Data Availability Statement. Data sharing is not applicable to this article as no new data were created or analyzed in this study.

Acknowledgement. I would like to acknowledge the guidance and contribution of Prof. Prasanta Panigrahi, IISER-Kolkata. This work was supported by the Trinity Barlow Scholarship and Nehru Bursary - Cambridge, and the Homi Bhabha Centre for Science Education, Tata Institute of Fundamental Research, Mumbai, India. The author acknowledges Prof. V. Singh of HBCSE for being a part of this effort.
[1] R. Horodecki, P. Horodecki, M. Horodecki, and K. Horodecki, Quantum entanglement, Reviews of modern physics 81, 865 (2009).

[2] J. Barrett, N. Linden, S. Massar, S. Pironio, S. Popescu, and D. Roberts, Nonlocal correlations as an informationtheoretic resource, Physical Review A 71, 022101 (2005).

[3] C. H. Bennett and D. P. DiVincenzo, Quantum information and computation, nature 404, 247 (2000).

[4] S.-B. Zheng and G.-C. Guo, Efficient scheme for twoatom entanglement and quantum information processing in cavity qed, Physical Review Letters 85, 2392 (2000).

[5] R. Laflamme, D. G. Cory, C. Negrevergne, and L. Viola, Nmr quantum information processing and entanglement, arXiv preprint quant-ph/0110029 (2001).

[6] J. H. Reina, L. Quiroga, and N. F. Johnson, Quantum entanglement and information processing via excitons in optically driven quantum dots, Physical Review A 62, 012305 (2000).

[7] M. Kues, C. Reimer, P. Roztocki, L. R. Cortés, S. Sciara, B. Wetzel, Y. Zhang, A. Cino, S. T. Chu, B. E. Little, et al., On-chip generation of high-dimensional entangled quantum states and their coherent control, Nature 546, 622 (2017).

[8] U. Khalid, J. ur Rehman, and H. Shin, Measurementbased quantum correlations for quantum information processing, Scientific Reports 10, 1 (2020).

[9] M. Guha Majumdar, Quantum information processing using the exchange interaction, Journal of Quantum Information Science 08, 139 (2018).
[10] M. G. Majumdar and P. Panigrahi, Teleportation and multipartite quantum state sharing using a seven qubit genuinely entangled state, arXiv preprint arXiv:1412.5102 (2014).

[11] D. Saha and P. K. Panigrahi, N-qubit quantum teleportation, information splitting and superdense coding through the composite ghz-bell channel, Quantum Information Processing 11, 615 (2012).

[12] M.-J. Zhao, Z.-G. Li, X. Li-Jost, and S.-M. Fei, Multiqubit quantum teleportation, Journal of Physics A: Mathematical and Theoretical 45, 405303 (2012).

[13] Z.-h. Zhang, L. Shu, and Z.-w. Mo, Quantum teleportation and superdense coding through the composite wbell channel, Quantum information processing 12, 1957 (2013).

[14] C. H. Bennett, G. Brassard, C. Crépeau, R. Jozsa, A. Peres, and W. K. Wootters, Teleporting an unknown quantum state via dual classical and einstein-podolskyrosen channels, Physical review letters 70, 1895 (1993).

[15] X. Gao, Z. Zhang, Y. Gong, B. Sheng, and X. Yu, Teleportation of entanglement using a three-particle entangled w state, JOSA B 34, 142 (2017).

[16] B.-S. Shi and A. Tomita, Teleportation of an unknown state by w state, Physics Letters A 296, 161 (2002).

[17] W. Tian-Yin and W. Qiao-Yan, Controlled quantum teleportation with bell states, Chinese Physics B 20, 040307 (2011).

[18] X. Tan, X. Li, and P. Yang, Perfect quantum teleportation via bell states, Computers, Materials \& Continua 
57, 495 (2018)

[19] J. Dong and J. Teng, Controlled teleportation of an arbitrary n-qudit state using nonmaximally entangled ghz states, The European Physical Journal D 49, 129 (2008).

[20] S. Hassanpour and M. Houshmand, Bidirectional teleportation of a pure epr state by using ghz states, Quantum Information Processing 15, 905 (2016).

[21] P. Espoukeh and P. Pedram, Quantum teleportation through noisy channels with multi-qubit ghz states, Quantum information processing 13, 1789 (2014).

[22] R.-G. Zhou, C. Qian, and H. Ian, Cyclic and bidirectional quantum teleportation via pseudo multi-qubit states, IEEE Access 7, 42445 (2019).

[23] A. Kumar, S. Adhikari, S. Banerjee, and S. Roy, Optimal quantum communication using multiparticle partially entangled states, Physical Review A 87, 022307 (2013).

[24] X.-Z. Zhou, X.-T. Yu, and Z.-C. Zhang, Multi-hop teleportation of an unknown qubit state based on w states, International Journal of Theoretical Physics 57, 981 (2018).

[25] D. Joy and M. Sabir, Efficient schemes for the quantum teleportation of a sub-class of tripartite entangled states, Quantum Information Processing 17, 170 (2018).

[26] Q. Quan, M.-J. Zhao, S.-M. Fei, H. Fan, W.-L. Yang, and G.-L. Long, Two-copy quantum teleportation, Scientific reports 8, 1 (2018).

[27] S. Rajiuddin, A. Baishya, B. K. Behera, and P. K. Panigrahi, Experimental realization of quantum teleportation of an arbitrary two-qubit state using a four-qubit cluster state, Quantum Information Processing 19, 87 (2020).

[28] Y.-H. Luo, H.-S. Zhong, M. Erhard, X.-L. Wang, L.-C. Peng, M. Krenn, X. Jiang, L. Li, N.-L. Liu, C.-Y. Lu, et al., Quantum teleportation in high dimensions, Physical review letters 123, 070505 (2019).

[29] K. Hofmann, A. Semenov, W. Vogel, and M. Bohmann, Quantum teleportation through atmospheric channels, Physica Scripta 94, 125104 (2019).

[30] F.-G. Deng, C.-Y. Li, Y.-S. Li, H.-Y. Zhou, and Y. Wang, Symmetric multiparty-controlled teleportation of an arbitrary two-particle entanglement, Physical Review A 72, 022338 (2005).

[31] F. Yan and D. Wang, Probabilistic and controlled teleportation of unknown quantum states, Physics Letters A 316, 297 (2003)

[32] X.-W. Zha, Z.-C. Zou, J.-X. Qi, and H.-Y. Song, Bidirectional quantum controlled teleportation via five-qubit cluster state, International Journal of Theoretical Physics 52, 1740 (2013).

[33] V. Sharma, C. Shukla, S. Banerjee, and A. Pathak, Controlled bidirectional remote state preparation in noisy environment: a generalized view, Quantum Information Processing 14, 3441 (2015).

[34] Y.-J. Duan, X.-W. Zha, X.-M. Sun, and J.-F. Xia, Bidirectional quantum controlled teleportation via a maximally seven-qubit entangled state, International Journal of Theoretical Physics 53, 2697 (2014).

[35] C. Shukla, A. Banerjee, and A. Pathak, Bidirectional controlled teleportation by using 5-qubit states: a generalized view, International Journal of Theoretical Physics 52, 3790 (2013).

[36] M. Hillery, V. Bužek, and A. Berthiaume, Quantum secret sharing, Physical Review A 59, 1829 (1999).

[37] L. Xiao, G. L. Long, F.-G. Deng, and J.-W. Pan, Efficient multiparty quantum-secret-sharing schemes, Phys- ical Review A 69, 052307 (2004).

[38] D. Gottesman, Theory of quantum secret sharing, Physical Review A 61, 042311 (2000).

[39] Z.-j. Zhang and C.-Y. Cheung, Shared quantum remote control: quantum operation sharing, Journal of Physics B: Atomic, Molecular and Optical Physics 44, 165508 (2011).

[40] Q. Ji, Y. Liu, X. Yin, X. Liu, and Z. Zhang, Quantum operation sharing with symmetric and asymmetric w states, Quantum information processing 12, 2453 (2013).

[41] J. Heo, C.-H. Hong, M.-S. Kang, H. Yang, H.-J. Yang, J.-P. Hong, and S.-G. Choi, Implementation of controlled quantum teleportation with an arbitrator for secure quantum channels via quantum dots inside optical cavities, Scientific reports 7, 1 (2017).

[42] T. Zheng, Y. Chang, and S.-B. Zhang, Arbitrated quantum signature scheme with quantum teleportation by using two three-qubit ghz states, Quantum Information Processing 19, 1 (2020).

[43] R.-J. Liu, M.-Q. Bai, F. Wu, and Y.-C. Zhang, Cycliccontrolled quantum operation teleportation in noisy environment, International Journal of Quantum Information 1950052 (2019).

[44] X. Wang, M. Byrd, and K. Jacobs, Numerical method for finding decoherence-free subspaces and its applications, Physical Review A 87, 012338 (2013).

[45] T. Van der Sar, Z. Wang, M. Blok, H. Bernien, T. Taminiau, D. Toyli, D. Lidar, D. Awschalom, R. Hanson, and V. Dobrovitski, Decoherence-protected quantum gates for a hybrid solid-state spin register, Nature 484, $82(2012)$

[46] M. Nakahara, Frank gaitan: Quantum error correction and fault tolerant quantum computing (2012).

[47] D. A. Lidar, I. L. Chuang, and K. B. Whaley, Decoherence-free subspaces for quantum computation, Physical Review Letters 81, 2594 (1998).

[48] İ. Yalçınkaya, B. Çakmak, G. Karpat, and F. Fanchini, Continuous dynamical decoupling and decoherence-free subspaces for qubits with tunable interaction, Quantum Information Processing 18, 156 (2019).

[49] R. Demkowicz-Dobrzański, J. Czajkowski, and P. Sekatski, Adaptive quantum metrology under general markovian noise, Physical Review X 7, 041009 (2017).

[50] G. Simon, C. Bruzewicz, K. Obenland, I. Chuang, R. Rines, J. Stuart, R. Niffenegger, J. Chiaverini, and J. Sage, Using fourier analysis and maximum likelihood estimation to identify and model non-markovian noise in quantum operations, in APS Meeting Abstracts (2019).

[51] A. W. Chin, S. F. Huelga, and M. B. Plenio, Quantum metrology in non-markovian environments, Physical review letters 109, 233601 (2012).

[52] T. Chanier, C. Pryor, and M. E. Flatte, Substitutional nickel impurities in diamond: Decoherence-free subspaces for quantum information processing, EPL (Europhysics Letters) 99, 67006 (2012).

[53] R. Ursin, F. Tiefenbacher, T. Schmitt-Manderbach, H. Weier, T. Scheidl, M. Lindenthal, B. Blauensteiner, T. Jennewein, J. Perdigues, P. Trojek, et al., Entanglement-based quantum communication over 144 km, Nature physics 3, 481 (2007).

[54] J.-W. Pan, C. Simon, Č. Brukner, and A. Zeilinger, Entanglement purification for quantum communication, $\mathrm{Na}-$ 
ture 410, 1067 (2001).

[55] A. V. Sergienko, Quantum communications and cryptography (CRC press, 2018).

[56] W. Tittel, How to overcome the distance barrier in quantum communication: Quantum repeaters and quantum memory, in CLEO: Applications and Technology (Optical Society of America, 2012) pp. JTh1K-1.

[57] M. Zwerger, W. Dür, and H. Briegel, Measurementbased quantum repeaters, Physical Review A 85, 062326 (2012).

[58] E. Brion, F. Carlier, V. Akulin, and K. Mølmer, Quantum repeater with rydberg-blocked atomic ensembles in fiber-coupled cavities, Physical Review A 85, 042324 (2012).

[59] J. Lee, H. Min, and S. D. Oh, Multipartite entanglement for entanglement teleportation, Physical Review A 66, 052318 (2002).

[60] M. Walter, D. Gross, and J. Eisert, Multipartite entanglement, Quantum Information: From Foundations to Quantum Technology Applications , 293 (2016).

[61] T. Dash, R. Sk, and P. K. Panigrahi, Deterministic joint remote state preparation of arbitrary two-qubit state through noisy cluster-ghz channel, Optics Communications 464, 125518 (2020).

[62] C. Di Franco, M. Paternostro, and M. Kim, Nested entangled states for distributed quantum channels, Physical Review A 77, 020303 (2008).

[63] M. Christandl, N. Datta, A. Ekert, and A. J. Landahl, Perfect state transfer in quantum spin networks, Physical review letters 92, 187902 (2004).

[64] M. Christandl, N. Datta, T. C. Dorlas, A. Ekert, A. Kay, and A. J. Landahl, Perfect transfer of arbitrary states in quantum spin networks, Physical Review A 71, 032312 (2005).

[65] D. Saha and P. K. Panigrahi, N-qubit quantum teleportation, information splitting and superdense coding through the composite ghz-bell channel, Quantum Information Processing 11, 615 (2012).

[66] X.-W. Zha, H.-Y. Song, J.-X. Qi, D. Wang, and Q. Lan, A genuine maximally seven-qubit entangled state, arXiv preprint arXiv:1110.5011 (2011).

[67] I. D. Brown, S. Stepney, A. Sudbery, and S. L. Braunstein, Searching for highly entangled multi-qubit states, Journal of Physics A: Mathematical and General 38, 1119 (2005).

[68] A. Borras, A. Plastino, J. Batle, C. Zander, M. Casas, and A. Plastino, Multiqubit systems: highly entangled states and entanglement distribution, Journal of Physics A: Mathematical and Theoretical 40, 13407 (2007).

[69] Y. Chen, Bidirectional controlled quantum teleportation by using five-qubit entangled state, International Journal of Theoretical Physics 53, 1454 (2014).

[70] X. Tan, X. Zhang, and T. Song, Deterministic quantum teleportation of a particular six-qubit state using sixqubit cluster state, International Journal of Theoretical Physics 55, 155 (2016).

[71] R.-G. Zhou, R. Xu, and H. Lan, Bidirectional quantum teleportation by using six-qubit cluster state, IEEE Access 7, 44269 (2019).

[72] M.-h. Sang, Bidirectional quantum controlled teleportation by using a seven-qubit entangled state, International Journal of Theoretical Physics 55, 380 (2016).

[73] W.-q. Hong, Asymmetric bidirectional controlled teleportation by using a seven-qubit entangled state, International Journal of Theoretical Physics 55, 384 (2016).

[74] M. S. S. Zadeh, M. Houshmand, and H. Aghababa, Bidirectional teleportation of a two-qubit state by using eight-qubit entangled state as a quantum channel, International Journal of Theoretical Physics 56, 2101 (2017).

[75] Z. Du, X. Li, and X. Liu, Bidirectional quantum teleportation with ghz states and epr pairs via entanglement swapping, International Journal of Theoretical Physics 59, 622 (2020). 


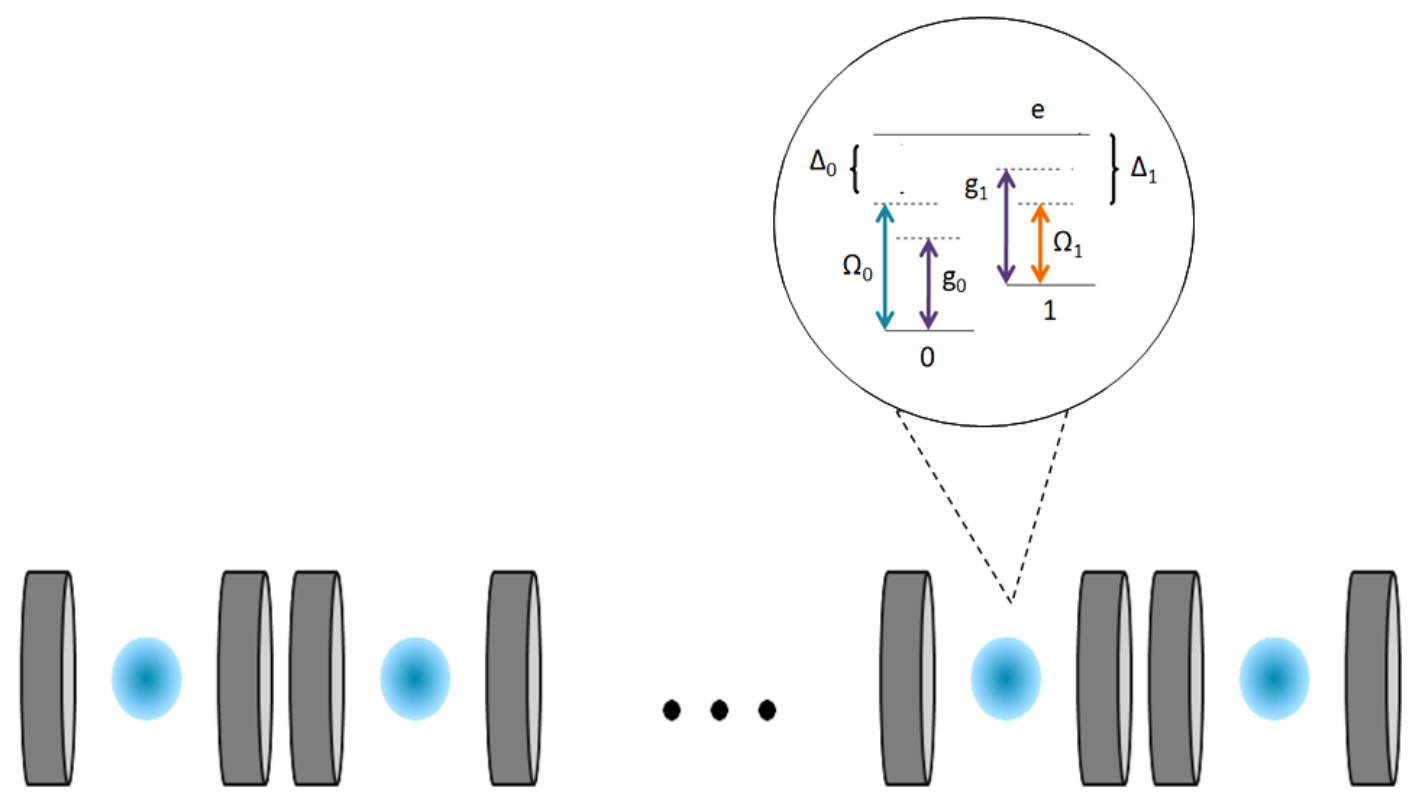

FIG. 1. Scheme for the generation of Matryoshka GHZ-Bell resource-states, where the effective spin-spin XY Hamiltonianan is obtained as an effective adiabatic Hamiltonian for a linear chain of optical cavities with each interacting with a three-level atomic system and that are mutually coupled via photon-hopping terms. The ground states of each atomic unit provide the computational space of each spin, and the dipole-forbidden transition between these states is realized as an (adiabatic) Raman transition through the excited state: $|e\rangle_{i}$ with $i=1,2, \ldots, N$. The cavity field drives off-resonantly the dipole-allowed channel $|j\rangle_{i} \leftrightarrow|e\rangle_{i}$ with the Rabi frequency $g_{j}, j=0,1$. Two lasers are also coupled to these atomic transitions with strength $\Omega_{j}$ and detuning $\Lambda_{j}$. If we assume large detunings, the excited level of each atomic unit as well as the corresponding cavity field's degrees of freedom can be eliminated adiabatically to give an effective spin-spin Hamiltonian

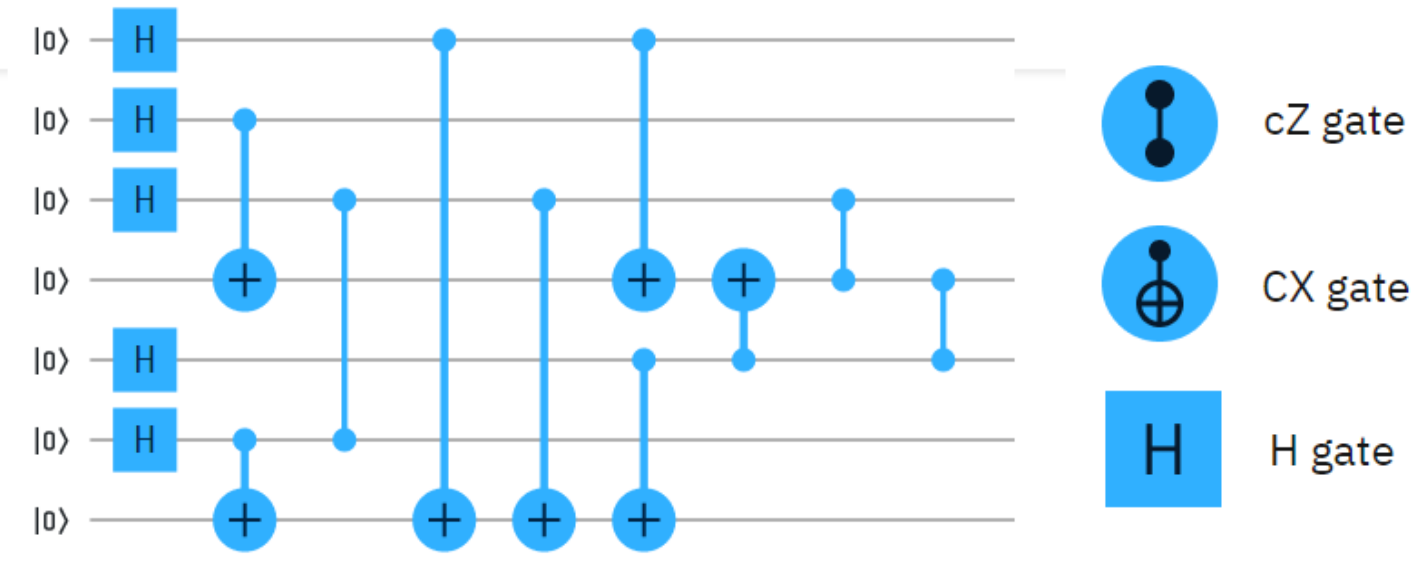

FIG. 2. Quantum Circuit for the generation of the seven-qubit genuinely entangled state, on IBM Quantum Experience. Here $C X$ gate is the CNOT gate, $c Z$ gate is the CPHASE gate and $H$ gate is the Hadamard gate 


\section{Supplementary Materials}

The teleportation of an arbitrary three-qubit state using our resource-state has as the initial composite state,

$$
\begin{aligned}
& \left|\Gamma_{7}^{(3)}\right\rangle=\left|\psi^{(3)}\right\rangle \otimes\left|\Gamma_{7}\right\rangle \\
& =a A_{000}|000\rangle+a A_{001}|001\rangle+a A_{010}|010\rangle+a A_{011}|011\rangle \\
& +a A_{100}|100\rangle+a A_{101}|101\rangle+a A_{110}|110\rangle+a A_{111}|111\rangle \\
& +b B_{000}|000\rangle+b B_{001}|001\rangle+b B_{010}|010\rangle+b B_{011}|011\rangle \\
& +b B_{100}|100\rangle+b B_{101}|101\rangle+b B_{110}|110\rangle+b B_{111}|111\rangle \\
& +c C_{000}|000\rangle+c C_{001}|001\rangle+c C_{010}|010\rangle+c C_{011}|011\rangle \\
& +c C_{100}|100\rangle+c C_{101}|101\rangle+c C_{110}|110\rangle+c C_{111}|111\rangle \\
& +d D_{000}|000\rangle+d D_{001}|001\rangle+d D_{010}|010\rangle+d D_{011}|011\rangle \\
& +d D_{100}|100\rangle+d D_{101}|101\rangle+d D_{110}|110\rangle+d D_{111}|111\rangle \\
& +e E_{000}|000\rangle+e E_{001}|001\rangle+e E_{010}|010\rangle+e E_{011}|011\rangle \\
& +e E_{100}|100\rangle+e E_{101}|101\rangle+e E_{110}|110\rangle+e E_{111}|111\rangle \\
& +f F_{000}|000\rangle+f F_{001}|001\rangle+f F_{010}|010\rangle+f F_{011}|011\rangle \\
& +f F_{100}|100\rangle+f F_{101}|101\rangle+f F_{110}|110\rangle+f B_{111}|111\rangle \\
& +g G_{000}|000\rangle+g G_{001}|001\rangle+g G_{010}|010\rangle+g G_{011}|011\rangle \\
& +g G_{100}|100\rangle+g G_{101}|101\rangle+g G_{110}|110\rangle+g G_{111}|111\rangle \\
& +h H_{000}|000\rangle+h H_{001}|001\rangle+h H_{010}|010\rangle+h H_{011}|011\rangle \\
& +h H_{100}|100\rangle+h H_{101}|101\rangle+h H_{110}|110\rangle+h H_{111}|111\rangle
\end{aligned}
$$

\section{THREE QUBIT STATES}

$\left|A_{000}\right\rangle=|0000000\rangle+|0000101\rangle-|0001011\rangle+|0001110\rangle$ $\left|A_{001}\right\rangle=|0000010\rangle+|0001001\rangle+|0001100\rangle-|0000111\rangle$ $\left|A_{010}\right\rangle=|0000111\rangle-|0000010\rangle+|0001001\rangle+|0001100\rangle$ $\left|A_{011}\right\rangle=|0000000\rangle+|0000101\rangle+|0001011\rangle-|0001110\rangle$ $\left|A_{100}\right\rangle=|0000011\rangle+|0000110\rangle-|0001000\rangle+|0001101\rangle$ $\left|A_{101}\right\rangle=|0000001\rangle-|0000100\rangle+|0001010\rangle+|0001111\rangle$ $\left|A_{110}\right\rangle=|0000001\rangle-|0000100\rangle-|0001010\rangle-|0001111\rangle$ $\left|A_{111}\right\rangle=|0001101\rangle-|0000011\rangle-|0000110\rangle-|0001000\rangle$

$\left|B_{000}\right\rangle=|0010000\rangle+|0010101\rangle-|0011011\rangle+|0011110\rangle$ $\left|B_{001}\right\rangle=|0010010\rangle+|0011001\rangle+|0011100\rangle-|0010111\rangle$ $\left|B_{010}\right\rangle=|0010111\rangle-|0010010\rangle+|0011001\rangle+|0011100\rangle$ $\left|B_{011}\right\rangle=|0010000\rangle+|0010101\rangle+|0011011\rangle-|0011110\rangle$ $\left|B_{100}\right\rangle=|0010011\rangle+|0010110\rangle-|0011000\rangle+|0011101\rangle$ $\left|B_{101}\right\rangle=|0010001\rangle-|0010100\rangle+|0011010\rangle+|0011111\rangle$ $\left|B_{110}\right\rangle=|0010001\rangle-|0010100\rangle-|0011010\rangle-|0011111\rangle$ $\left|B_{111}\right\rangle=|0011101\rangle-|0010011\rangle-|0010110\rangle-|0011000\rangle$

$\left|C_{000}\right\rangle=|0100000\rangle+|0100101\rangle-|0101011\rangle+|0101110\rangle$ $\left|C_{001}\right\rangle=|0100010\rangle+|0101001\rangle+|0101100\rangle-|0100111\rangle$ $\left|C_{010}\right\rangle=|0100111\rangle-|0100010\rangle+|0101001\rangle+|0101100\rangle$ $\left|C_{011}\right\rangle=|0100000\rangle+|0100101\rangle+|0101011\rangle-|0101110\rangle$ $\left|C_{100}\right\rangle=|0100011\rangle+|0100110\rangle-|0101000\rangle+|0101101\rangle$
$\left|C_{101}\right\rangle=|0100001\rangle-|0100100\rangle+|0101010\rangle+|0101111\rangle$ $\left|C_{110}\right\rangle=|0100001\rangle-|0100100\rangle-|0101010\rangle-|0101111\rangle$ $\left|C_{111}\right\rangle=|0101101\rangle-|0100011\rangle-|0100110\rangle-|0101000\rangle$

$\left|D_{000}\right\rangle=|0110000\rangle+|0110101\rangle-|0111011\rangle+|0111110\rangle$

$\left|D_{001}\right\rangle=|0110010\rangle+|0111001\rangle+|0111100\rangle-|0110111\rangle$

$\left|D_{010}\right\rangle=|0110111\rangle-|0110010\rangle+|0111001\rangle+|0111100\rangle$

$\left|D_{011}\right\rangle=|0110000\rangle+|0110101\rangle+|0111011\rangle-|0111110\rangle$

$\left|D_{100}\right\rangle=|0110011\rangle+|0110110\rangle-|0111000\rangle+|0111101\rangle$

$\left|D_{101}\right\rangle=|0110001\rangle-|0110100\rangle+|0111010\rangle+|0111111\rangle$

$\left|D_{110}\right\rangle=|0110001\rangle-|0110100\rangle-|0111010\rangle-|0111111\rangle$

$\left|D_{111}\right\rangle=|0111101\rangle-|0110011\rangle-|0110110\rangle-|0111000\rangle$

$\left|E_{000}\right\rangle=|1000000\rangle+|1000101\rangle-|1001011\rangle+|1001110\rangle$ $\left|E_{001}\right\rangle=|1000010\rangle+|1001001\rangle+|1001100\rangle-|1000111\rangle$ $\left|E_{010}\right\rangle=|1000111\rangle-|1000010\rangle+|0001001\rangle+|0001100\rangle$ $\left|E_{011}\right\rangle=|1000000\rangle+|1000101\rangle+|1001011\rangle-|1001110\rangle$ $\left|E_{100}\right\rangle=|1000011\rangle+|1000110\rangle-|1001000\rangle+|1001101\rangle$ $\left|E_{101}\right\rangle=|1000001\rangle-|1000100\rangle+|1001010\rangle+|1001111\rangle$ $\left|E_{110}\right\rangle=|1000001\rangle-|1000100\rangle-|1001010\rangle-|1001111\rangle$ $\left|E_{111}\right\rangle=|1001101\rangle-|1000011\rangle-|1000110\rangle-|1001000\rangle$

$\left|F_{000}\right\rangle=|1010000\rangle+|1010101\rangle-|1011011\rangle+|1011110\rangle$

$\left|F_{001}\right\rangle=|1010010\rangle+|1011001\rangle+|1011100\rangle-|1010111\rangle$

$\left|F_{010}\right\rangle=|1010111\rangle-|1010010\rangle+|1011001\rangle+|1011100\rangle$

$\left|F_{011}\right\rangle=|1010000\rangle+|1010101\rangle+|1011011\rangle-|1011110\rangle$

$\left|F_{100}\right\rangle=|1010011\rangle+|1010110\rangle-|1011000\rangle+|1011101\rangle$

$\left|F_{101}\right\rangle=|1010001\rangle-|1010100\rangle+|1011010\rangle+|1011111\rangle$

$\left|F_{110}\right\rangle=|1010001\rangle-|1010100\rangle-|1011010\rangle-|1011111\rangle$

$\left|F_{111}\right\rangle=|1011101\rangle-|1010011\rangle-|1010110\rangle-|1011000\rangle$

$\left|G_{000}\right\rangle=|1100000\rangle+|1100101\rangle-|1101011\rangle+|1101110\rangle$

$\left|G_{001}\right\rangle=|1100010\rangle+|1101001\rangle+|1101100\rangle-|1100111\rangle$

$\left|G_{010}\right\rangle=|1100111\rangle-|1100010\rangle+|1101001\rangle+|1101100\rangle$

$\left|G_{011}\right\rangle=|1100000\rangle+|1100101\rangle+|1101011\rangle-|1101110\rangle$

$\left|G_{100}\right\rangle=|1100011\rangle+|1100110\rangle-|1101000\rangle+|1101101\rangle$

$\left|G_{101}\right\rangle=|1100001\rangle-|1100100\rangle+|1101010\rangle+|1101111\rangle$

$\left|G_{110}\right\rangle=|1100001\rangle-|1100100\rangle-|1101010\rangle-|1101111\rangle$

$\left|G_{111}\right\rangle=|1101101\rangle-|1100011\rangle-|0000110\rangle-|0001000\rangle$

$\left|H_{000}\right\rangle=|1110000\rangle+|1110101\rangle-|1111011\rangle+|1111110\rangle$

$\left|H_{001}\right\rangle=|1110010\rangle+|1111001\rangle+|1111100\rangle-|1110111\rangle$

$\left|H_{010}\right\rangle=|1110111\rangle-|1110010\rangle+|1111001\rangle+|1111100\rangle$

$\left|H_{011}\right\rangle=|1110000\rangle+|1110101\rangle+|1111011\rangle-|1111110\rangle$

$\left|H_{100}\right\rangle=|1110011\rangle+|1110110\rangle-|1111000\rangle+|1111101\rangle$

$\left|H_{101}\right\rangle=|1110001\rangle-|1110100\rangle+|1111010\rangle+|1111111\rangle$

$\left|H_{110}\right\rangle=|1110001\rangle-|1110100\rangle-|1111010\rangle-|1111111\rangle$

$\left|H_{111}\right\rangle=|1111101\rangle-|1110011\rangle-|1110110\rangle-|1111000\rangle$

\section{DECOMPOSITION OF ARBITRARY THREE QUBIT STATE WITH $\left|\Psi_{7}\right\rangle$}

$$
\begin{array}{r}
(a|000\rangle+b|001\rangle+c|010\rangle+d|011\rangle+e|100\rangle \\
+f|101\rangle+g|110\rangle+h|111\rangle)\left|\Psi_{7}\right\rangle
\end{array}
$$




$$
\begin{gathered}
=\sum_{\text {permutations }}\left((-1)^{I_{1}} A_{a_{1} a_{2} a_{3}}+(-1)^{I_{2}} B_{b_{1} b_{2} b_{3}}\right. \\
+(-1)^{I_{3}} C_{c_{1} c_{2} c_{3}}+(-1)^{I_{4}} D_{d_{1} d_{2} d_{3}} \\
+(-1)^{I_{5}} E_{e_{1} e_{2} e_{3}} \\
+(-1)^{I_{6}} F_{f_{1} f_{2} f_{3}} \\
+(-1)^{I_{7}} G_{g_{1} g_{2} g_{3}}+(-1)^{I_{8}} H_{\left.h_{1} h_{2} h_{3}\right)} \\
\quad \\
\quad\left((-1)^{I_{1}} a\left|a_{1} a_{2} a_{3}\right\rangle+(-1)^{I_{2}} b\left|b_{1} b_{2} b_{3}\right\rangle\right. \\
+(-1)^{I_{3}} c\left|c_{1} c_{2} c_{3}\right\rangle+(-1)^{I_{4}} d\left|d_{1} d_{2} d_{3}\right\rangle \\
+(-1)^{I_{5}} e\left|e_{1} e_{2} e_{3}\right\rangle+(-1)^{I_{6}} f\left|f_{1} f_{2} f_{3}\right\rangle \\
\left.+(-1)^{I_{7}} g\left|g_{1} g_{2} g_{3}\right\rangle+(-1)^{I_{8}} h\left|h_{1} h_{2} h_{3}\right\rangle\right)
\end{gathered}
$$

where $I_{i}(\mathrm{i}=1,2,3,4,5,6,7,8)$ can take values 0 or 1 independently, and $L_{j}(\mathrm{~L}=\mathrm{a}, \mathrm{b}, \mathrm{c}, \mathrm{d}, \mathrm{e}, \mathrm{f}, \mathrm{g}, \mathrm{h} ; \mathrm{j}=1,2$, $3)$ can take values 0 or 1 independently. The summation is over all possible permutation states obtained.

\section{TRANSFORMATIONS FOR THREE-QUBIT TELEPORTATION}

Projection of $i^{\text {th }}$ component $P_{i}$ :

$$
P_{1}=\left(\begin{array}{ll}
1 & 0 \\
0 & 0
\end{array}\right), P_{2}=\left(\begin{array}{ll}
0 & 0 \\
0 & 1
\end{array}\right)
$$

Flip and Projection of $i^{\text {th }}$ component $F_{i}$ :

$$
F_{1}=\left(\begin{array}{ll}
0 & 1 \\
0 & 0
\end{array}\right), F_{2}=\left(\begin{array}{ll}
0 & 0 \\
1 & 0
\end{array}\right)
$$


State Obtained by Alice

$A_{000}+B_{001}+C_{010}+D_{011}+E_{100}+F_{101}+G_{110}+H_{111}$ $A_{000}-B_{001}+C_{010}+D_{011}+E_{100}+F_{101}+G_{110}+H_{111}$ $A_{000}+B_{001}+C_{010}-D_{011}+E_{100}+F_{101}+G_{110}+H_{111}$ $A_{000}+B_{001}+C_{010}+D_{011}+E_{100}-F_{101}+G_{110}+H_{111}$ $A_{000}+B_{001}+C_{010}+D_{011}+E_{100}+F_{101}+G_{110}-H_{111}$ $A_{000}-B_{001}+C_{010}-D_{011}+E_{100}+F_{101}+G_{110}+H_{111}$ $A_{000}-B_{001}+C_{010}+D_{011}+E_{100}-F_{101}+G_{110}+H_{111}$ $A_{000}-B_{001}+C_{010}-D_{011}+E_{100}-F_{101}+G_{110}+H_{111}$ $A_{000}+B_{001}+C_{010}-D_{011}+E_{100}-F_{101}+G_{110}+H_{111}$ $A_{000}-B_{001}+C_{010}+D_{011}+E_{100}+F_{101}+G_{110}-H_{111}$ $A_{000}+B_{001}+C_{010}-D_{011}+E_{100}+F_{101}+G_{110}-H_{111}$ $A_{000}+B_{001}+C_{010}+D_{011}+E_{100}-F_{101}+G_{110}-H_{111}$ $A_{000}-B_{001}+C_{010}+D_{011}+E_{100}-F_{101}+G_{110}-H_{111}$ $A_{000}+B_{001}+C_{010}+D_{011}+E_{100}-F_{101}+G_{110}-H_{111}$ $A_{000}-B_{001}+C_{010}-D_{011}+E_{100}+F_{101}+G_{110}-H_{111}$ $A_{000}-B_{001}+C_{010}-D_{011}+E_{100}-F_{101}+G_{110}-H_{111}$ $A_{000}+B_{001}-C_{010}+D_{011}+E_{100}+F_{101}+G_{110}+H_{111}$ $A_{000}+B_{001}-C_{010}+D_{011}+E_{100}+F_{101}-G_{110}+H_{111}$ $A_{000}+B_{001}-C_{010}-D_{011}+E_{100}+F_{101}+G_{110}+H_{111}$ $A_{000}+B_{001}-C_{010}+D_{011}+E_{100}+F_{101}-G_{110}+H_{111}$ $A_{000}+B_{001}-C_{010}+D_{011}+E_{100}+F_{101}+G_{110}-H_{111}$ $A_{000}+B_{001}+C_{010}-D_{011}+E_{100}+F_{101}-G_{110}+H_{111}$ $A_{000}+B_{001}+C_{010}+D_{011}+E_{100}+F_{101}-G_{110}-H_{111}$ $A_{000}+B_{001}-C_{010}-D_{011}+E_{100}+F_{101}-G_{110}+H_{111}$ $A_{000}+B_{001}-C_{010}-D_{011}+E_{100}+F_{101}+G_{110}-H_{111}$ $A_{000}+B_{001}+C_{010}-D_{011}+E_{100}+F_{101}-G_{110}-H_{111}$ $A_{000}+B_{001}-C_{010}+D_{011}+E_{100}+F_{101}-G_{110}-H_{111}$ $A_{000}+B_{001}-C_{010}-D_{011}+E_{100}+F_{101}-G_{110}-H_{111}$ $A_{000}+B_{001}+C_{010}+D_{011}-E_{100}+F_{101}+G_{110}+H_{111}$ $A_{000}+B_{001}-C_{010}+D_{011}-E_{100}-F_{101}+G_{110}+H_{111}$ $A_{000}+B_{001}+C_{010}+D_{011}-E_{100}-F_{101}+G_{110}+H_{111}$ $A_{000}+B_{001}+C_{010}+D_{011}-E_{100}+F_{101}+G_{110}-H_{111}$ $A_{000}+B_{001}+C_{010}+D_{011}+E_{100}-F_{101}-G_{110}+H_{111}$ $A_{000}+B_{001}+C_{010}+D_{011}+E_{100}+F_{101}-G_{110}-H_{111}$ $A_{000}+B_{001}+C_{010}+D_{011}+E_{100}-F_{101}-G_{110}+H_{111}$ $A_{000}+B_{001}+C_{010}+D_{011}-E_{100}+F_{101}-G_{110}-H_{111}$ $A_{000}+B_{001}+C_{010}+D_{011}+E_{100}-F_{101}-G_{110}-H_{111}$ $A_{000}+B_{001}+C_{010}+D_{011}-E_{100}-F_{101}-G_{110}-H_{111}$ $A_{001}+B_{000}+C_{011}+D_{010}+E_{101}+F_{100}+G_{111}+H_{110}$ $-A_{001}+B_{000}+C_{011}+D_{010}+E_{101}+F_{100}+G_{111}+H_{110}$ $A_{001}+B_{000}-C_{011}+D_{010}+E_{101}+F_{100}+G_{111}+H_{110}$ $A_{001}+B_{000}+C_{011}+D_{010}-E_{101}+F_{100}+G_{111}+H_{110}$ $A_{001}+B_{000}+C_{011}+D_{010}+E_{101}+F_{100}-G_{111}+H_{110}$ $-A_{001}+B_{000}-C_{011}+D_{010}+E_{101}+F_{100}+G_{111}+H_{110}$ $-A_{001}+B_{000}+C_{011}+D_{010}-E_{101}+F_{100}+G_{111}+H_{110}$ $-A_{001}+B_{000}+C_{011}+D_{010}+E_{101}+F_{100}-G_{111}+H_{110}$ $A_{001}+B_{000}-C_{011}+D_{010}-E_{101}+F_{100}+G_{111}+H_{110}$ $A_{001}+B_{000}-C_{011}+D_{010}+E_{101}+F_{100}-G_{111}+H_{110}$ $A_{001}+B_{000}+C_{011}+D_{010}-E_{101}+F_{100}-G_{111}+H_{110}$ $-A_{001}+B_{000}-C_{011}+D_{010}-E_{101}+F_{100}+G_{111}+H_{110}$ $-A_{001}+B_{000}+C_{011}+D_{010}-E_{101}+F_{100}-G_{111}+H_{110}$ $-A_{001}+B_{000}-C_{011}+D_{010}+E_{101}+F_{100}-G_{111}+H_{110}$ $A_{001}+B_{000}-C_{011}+D_{010}-E_{101}+F_{100}-G_{111}+H_{110}$ $-A_{001}+B_{000}-C_{011}+D_{010}-E_{101}+F_{100}-G_{111}+H_{110}$ $A_{001}+B_{000}+C_{011}-D_{010}+E_{101}+F_{100}+G_{111}+H_{110}$ $A_{001}+B_{000}+C_{011}+D_{010}+E_{101}+F_{100}+G_{111}-H_{110}$ $A_{001}+B_{000}-C_{011}+D_{010}+E_{101}+F_{100}+G_{111}-H_{110}$ $A_{001}+B_{000}-C_{011}-D_{010}+E_{101}+F_{100}+G_{111}+H_{110}$ $A_{001}+B_{000}+C_{011}-D_{010}+E_{101}+F_{100}-G_{111}+H_{110}$ $A_{001}+B_{000}+C_{011}-D_{010}+E_{101}+F_{100}+G_{111}-H_{110}$
Short-Hand Form of Transformation

$I_{2} \otimes I_{2} \otimes I_{2}$

$I_{2} \otimes I_{2} \otimes P_{2}+\sigma_{z} \otimes P_{1} \otimes P_{1}+I_{2} \otimes P_{2} \otimes P_{1}$

$I_{2} \otimes I_{2} \otimes P_{2}+I_{2} \otimes P_{1} \otimes P_{1}+\sigma_{z} \otimes P_{2} \otimes P_{1}$

$I_{2} \otimes I_{2} \otimes P_{1}+\sigma_{z} \otimes P_{1} \otimes P_{2}+I_{2} \otimes P_{2} \otimes P_{2}$

$I_{2} \otimes I_{2} \otimes P_{1}+\sigma_{z} \otimes P_{2} \otimes P_{2}+I_{2} \otimes P_{2} \otimes P_{2}$

$I_{2} \otimes I_{2} \otimes P_{2}+\sigma_{z} \otimes P_{1} \otimes P_{1}+\sigma_{z} \otimes P_{2} \otimes P_{1}$

$\sigma_{z} \otimes P_{1} \otimes P_{1}+I_{2} \otimes P_{2} \otimes P_{1}+\sigma_{z} \otimes P_{1} \otimes P_{2}+I_{2} \otimes P_{2} \otimes P_{2}$

$\sigma_{z} \otimes P_{1} \otimes P_{1}+\sigma_{z} \otimes P_{2} \otimes P_{1}+\sigma_{z} \otimes P_{1} \otimes P_{2}+I_{2} \otimes P_{2} \otimes P_{2}$

$I_{2} \otimes P_{1} \otimes P_{1}+\sigma_{z} \otimes P_{2} \otimes P_{1}+\sigma_{z} \otimes P_{1} \otimes P_{2}+I_{2} \otimes P_{2} \otimes P_{2}$

$\sigma_{z} \otimes P_{1} \otimes P_{1}+I_{2} \otimes P_{2} \otimes P_{1}+P_{2} \otimes P_{1} \otimes P_{2}+\sigma_{z} \otimes P_{2} \otimes P_{2}$

$I_{2} \otimes P_{1} \otimes P_{1}+\sigma_{z} \otimes P_{2} \otimes P_{1}+I_{2} \otimes P_{1} \otimes P_{2}+\sigma_{z} \otimes P_{2} \otimes P_{2}$

$I_{2} \otimes P_{1} \otimes P_{1}+I_{2} \otimes P_{2} \otimes P_{1}+\sigma_{z} \otimes P_{1} \otimes P_{2}+\sigma_{z} \otimes P_{2} \otimes P_{2}$

$\sigma_{z} \otimes P_{1} \otimes P_{1}+I_{2} \otimes P_{2} \otimes P_{1}+\sigma_{z} \otimes P_{1} \otimes P_{2}+\sigma_{z} \otimes P_{2} \otimes P_{2}$ $I_{2} \otimes P_{1} \otimes P_{1}+I_{2} \otimes P_{2} \otimes P_{1}+\sigma_{z} \otimes P_{1} \otimes P_{2}+\sigma_{z} \otimes P_{2} \otimes P_{2}$ $\sigma_{z} \otimes P_{1} \otimes P_{1}+\sigma_{z} \otimes P_{2} \otimes P_{1}+I_{2} \otimes P_{1} \otimes P_{2}+\sigma_{z} \otimes P_{2} \otimes P_{2}$ $\sigma_{z} \otimes P_{1} \otimes P_{1}+\sigma_{z} \otimes P_{2} \otimes P_{1}+\sigma_{z} \otimes P_{1} \otimes P_{2}+\sigma_{z} \otimes P_{2} \otimes P_{2}$ $I_{2} \otimes P_{1} \otimes P_{1}-\sigma_{z} \otimes P_{2} \otimes P_{1}+I_{2} \otimes P_{1} \otimes P_{2}+I_{2} \otimes P_{2} \otimes P_{2}$ $I_{2} \otimes P_{1} \otimes P_{1}-\sigma_{z} \otimes P_{2} \otimes P_{1}+I_{2} \otimes P_{1} \otimes P_{2}-\sigma_{z} \otimes P_{2} \otimes P_{2}$ $I_{2} \otimes P_{1} \otimes P_{1}-I_{2} \otimes P_{2} \otimes P_{1}+I_{2} \otimes P_{1} \otimes P_{2}+I_{2} \otimes P_{2} \otimes P_{2}$ $I_{2} \otimes P_{1} \otimes P_{1}-\sigma_{z} \otimes P_{2} \otimes P_{1}+I_{2} \otimes P_{1} \otimes P_{2}-\sigma_{z} \otimes P_{2} \otimes P_{2}$ $I_{2} \otimes P_{1} \otimes P_{1}-\sigma_{z} \otimes P_{2} \otimes P_{1}+I_{2} \otimes P_{1} \otimes P_{2}+\sigma_{z} \otimes P_{2} \otimes P_{2}$ $I_{2} \otimes P_{1} \otimes P_{1}+\sigma_{z} \otimes P_{2} \otimes P_{1}+I_{2} \otimes P_{1} \otimes P_{2}+\sigma_{z} \otimes P_{2} \otimes P_{2}$ $I_{2} \otimes P_{1} \otimes P_{1}+I_{2} \otimes P_{2} \otimes P_{1}+I_{2} \otimes P_{1} \otimes P_{2}+\sigma_{z} \otimes P_{2} \otimes P_{2}$ $I_{2} \otimes P_{1} \otimes P_{1}-I_{2} \otimes P_{2} \otimes P_{1}+I_{2} \otimes P_{1} \otimes P_{2}-\sigma_{z} \otimes P_{2} \otimes P_{2}$ $I_{2} \otimes P_{1} \otimes P_{1}-I_{2} \otimes P_{2} \otimes P_{1}+I_{2} \otimes P_{1} \otimes P_{2}+\sigma_{z} \otimes P_{2} \otimes P_{2}$ $I_{2} \otimes P_{1} \otimes P_{1}+\sigma_{z} \otimes P_{2} \otimes P_{1}+I_{2} \otimes P_{1} \otimes P_{2}-I_{2} \otimes P_{2} \otimes P_{2}$ $I_{2} \otimes P_{1} \otimes P_{1}-\sigma_{z} \otimes P_{2} \otimes P_{1}+I_{2} \otimes P_{1} \otimes P_{2}-I_{2} \otimes P_{2} \otimes P_{2}$ $I_{2} \otimes P_{1} \otimes P_{1}-I_{2} \otimes P_{2} \otimes P_{1}+I_{2} \otimes P_{1} \otimes P_{2}-I_{2} \otimes P_{2} \otimes P_{2}$ $I_{2} \otimes P_{1} \otimes P_{1}+I_{2} \otimes P_{2} \otimes P_{1}-\sigma_{z} \otimes P_{1} \otimes P_{2}+I_{2} \otimes P_{2} \otimes P_{2}$ $\sigma_{z} \otimes P_{1} \otimes P_{1}-\sigma_{z} \otimes P_{2} \otimes P_{1}-I_{2} \otimes P_{1} \otimes P_{2}+I_{2} \otimes P_{2} \otimes P_{2}$ $I_{2} \otimes P_{1} \otimes P_{1}+I_{2} \otimes P_{2} \otimes P_{1}-I_{2} \otimes P_{1} \otimes P_{2}+I_{2} \otimes P_{2} \otimes P_{2}$ $I_{2} \otimes P_{1} \otimes P_{1}+I_{2} \otimes P_{2} \otimes P_{1}-\sigma_{z} \otimes P_{1} \otimes P_{2}+\sigma_{z} \otimes P_{2} \otimes P_{2}$ $I_{2} \otimes P_{1} \otimes P_{1}+I_{2} \otimes P_{2} \otimes P_{1}+\sigma_{z} \otimes P_{1} \otimes P_{2}-\sigma_{z} \otimes P_{2} \otimes P_{2}$ $I_{2} \otimes P_{1} \otimes P_{1}+I_{2} \otimes P_{2} \otimes P_{1}+I_{2} \otimes P_{1} \otimes P_{2}-I_{2} \otimes P_{2} \otimes P_{2}$ $I_{2} \otimes P_{1} \otimes P_{1}+I_{2} \otimes P_{2} \otimes P_{1}+\sigma_{z} \otimes P_{1} \otimes P_{2}-\sigma_{z} \otimes P_{2} \otimes P_{2}$ $I_{2} \otimes P_{1} \otimes P_{1}+I_{2} \otimes P_{2} \otimes P_{1}-\sigma_{z} \otimes P_{1} \otimes P_{2}-I_{2} \otimes P_{2} \otimes P_{2}$ $I_{2} \otimes P_{1} \otimes P_{1}+I_{2} \otimes P_{2} \otimes P_{1}+\sigma_{z} \otimes P_{1} \otimes P_{2}-I_{2} \otimes P_{2} \otimes P_{2}$ $I_{2} \otimes P_{1} \otimes P_{1}+I_{2} \otimes P_{2} \otimes P_{1}-I_{2} \otimes P_{1} \otimes P_{2}-I_{2} \otimes P_{2} \otimes P_{2}$ $\sigma_{x} \otimes I_{2} \otimes I_{2}$ $\sigma_{x} \otimes P_{1} \otimes P_{2}+\sigma_{x} \otimes P_{2} \otimes P_{2}+I \sigma_{y} \otimes P_{1} \otimes P_{1}$ $\sigma_{x} \otimes P_{1} \otimes I_{2}+I \sigma_{y} \otimes P_{2} \otimes P_{1}+\sigma_{x} \otimes I_{2} \otimes P_{2}$ $\sigma_{x} \otimes I_{2} \otimes P_{1}+I \sigma_{y} \otimes P_{1} \otimes P_{2}+\sigma_{x} \otimes P_{2} \otimes P_{2}$ $\sigma_{x} \otimes I_{2} \otimes P_{1}+\sigma_{x} \otimes P_{1} \otimes P_{2}+I \sigma_{y} \otimes P_{2} \otimes P_{2}$ $I \sigma_{y} \otimes I_{2} \otimes P_{1}+\sigma_{x} \otimes I_{2} \otimes P_{2}$ $I \sigma_{y} \otimes P_{1} \otimes P_{1}+\sigma_{x} \otimes P_{2} \otimes P_{1}+I \sigma_{y} \otimes P_{1} \otimes P_{2}+\sigma_{x} \otimes P_{2} \otimes P_{2}$ $I \sigma_{y} \otimes P_{1} \otimes P_{1}+\sigma_{x} \otimes P_{2} \otimes P_{1}+\sigma_{x} \otimes P_{1} \otimes P_{2}+I \sigma_{y} \otimes P_{2} \otimes P_{2}$ $\sigma_{x} \otimes P_{1} \otimes P_{1}+I \sigma_{y} \otimes P_{2} \otimes P_{1}+I \sigma_{y} \otimes P_{1} \otimes P_{2}+\sigma_{x} \otimes P_{2} \otimes P_{2}$ $\sigma_{x} \otimes P_{1} \otimes P_{1}+I \sigma_{y} \otimes P_{2} \otimes P_{1}+\sigma_{x} \otimes P_{1} \otimes P_{2}+I \sigma_{y} \otimes P_{2} \otimes P_{2}$ $\sigma_{x} \otimes P_{1} \otimes P_{1}+\sigma_{x} \otimes P_{2} \otimes P_{1}+I \sigma_{y} \otimes P_{1} \otimes P_{2}+I \sigma_{y} \otimes P_{2} \otimes P_{2}$ $\sigma_{x} \otimes P_{1} \otimes P_{1}+I \sigma_{y} \otimes P_{2} \otimes P_{1}+I \sigma_{y} \otimes P_{1} \otimes P_{2}+\sigma_{x} \otimes P_{2} \otimes P_{2}$ $I \sigma_{y} \otimes P_{1} \otimes P_{1}+\sigma_{x} \otimes P_{2} \otimes P_{1}+I \sigma_{y} \otimes P_{1} \otimes P_{2}+I \sigma_{y} \otimes P_{2} \otimes P_{2}$ $I \sigma_{y} \otimes P_{1} \otimes P_{1}+I \sigma_{y} \otimes P_{2} \otimes P_{1}+\sigma_{x} \otimes P_{1} \otimes P_{2}+I \sigma_{y} \otimes P_{2} \otimes P_{2}$ $\sigma_{x} \otimes P_{1} \otimes P_{1}+I \sigma_{y} \otimes P_{2} \otimes P_{1}+I \sigma_{y} \otimes P_{1} \otimes P_{2}+I \sigma_{y} \otimes P_{2} \otimes P_{2}$ $I \sigma_{y} \otimes P_{1} \otimes P_{1}+I \sigma_{y} \otimes P_{2} \otimes P_{1}+\sigma_{x} \otimes P_{1} \otimes P_{2}+I \sigma_{y} \otimes P_{2} \otimes P_{2}$ $\sigma_{x} \otimes P_{1} \otimes P_{1}-I \sigma_{y} \otimes P_{2} \otimes P_{1}+\sigma_{x} \otimes P_{1} \otimes P_{2}+\sigma_{x} \otimes P_{2} \otimes P_{2}$ $\sigma_{x} \otimes P_{1} \otimes P_{1}+\sigma_{x} \otimes P_{2} \otimes P_{1}+\sigma_{x} \otimes P_{1} \otimes P_{2}-I \sigma_{y} \otimes P_{2} \otimes P_{2}$ $\sigma_{x} \otimes P_{1} \otimes P_{1}+I \sigma_{y} \otimes P_{2} \otimes P_{1}+\sigma_{x} \otimes P_{1} \otimes P_{2}+I \sigma_{y} \otimes P_{2} \otimes P_{2}$ $\sigma_{x} \otimes P_{1} \otimes P_{1}-\sigma_{x} \otimes P_{2} \otimes P_{1}+\sigma_{x} \otimes P_{1} \otimes P_{2}+\sigma_{x} \otimes P_{2} \otimes P_{2}$ $\sigma_{x} \otimes P_{1} \otimes P_{1}-I \sigma_{y} \otimes P_{2} \otimes P_{1}+\sigma_{x} \otimes P_{1} \otimes P_{2}+I \sigma_{y} \otimes P_{2} \otimes P_{2}$ $\sigma_{x} \otimes P_{1} \otimes P_{1}-I \sigma_{y} \otimes P_{2} \otimes P_{1}+\sigma_{x} \otimes P_{1} \otimes P_{2}-I \sigma_{y} \otimes P_{2} \otimes P_{2}$ 
$A_{001}+B_{000}+C_{011}+D_{010}+E_{101}+F_{100}-G_{111}-H_{110}$ $A_{001}+B_{000}-C_{011}-D_{010}+E_{101}+F_{100}-G_{111}+H_{110}$ $A_{001}+B_{000}-C_{011}-D_{010}+E_{101}+F_{100}+G_{111}-H_{110}$ $A_{001}+B_{000}-C_{011}+D_{010}+E_{101}+F_{100}-G_{111}-H_{110}$ $A_{001}+B_{000}+C_{011}-D_{010}+E_{101}+F_{100}-G_{111}-H_{110}$ $A_{001}+B_{000}-C_{011}-D_{010}+E_{101}+F_{100}-G_{111}-H_{110}$ $A_{001}+B_{000}+C_{011}+D_{010}+E_{101}-F_{100}+G_{111}+H_{110}$ $A_{001}+B_{000}+C_{011}+D_{010}-E_{101}-F_{100}+G_{111}+H_{110}$ $A_{001}+B_{000}+C_{011}+D_{010}-E_{101}+F_{100}-G_{111}+H_{110}$ $A_{001}+B_{000}+C_{011}+D_{010}-E_{101}+F_{100}+G_{111}-H_{110}$ $A_{001}+B_{000}+C_{011}+D_{010}+E_{101}-F_{100}-G_{111}+H_{110}$ $A_{001}+B_{000}+C_{011}+D_{010}+E_{101}-F_{100}+G_{111}-H_{110}$ $A_{001}+B_{000}+C_{011}+D_{010}-E_{101}-F_{100}-G_{111}+H_{110}$ $A_{001}+B_{000}+C_{011}+D_{010}-E_{101}+F_{100}-G_{111}-H_{110}$ $A_{001}+B_{000}+C_{011}+D_{010}+E_{101}-F_{100}-G_{111}-H_{110}$ $+A_{001}+B_{000}+C_{011}+D_{010}-E_{101}-F_{100}-G_{111}-H_{110}$ $A_{010}-B_{011}+C_{000}+D_{001}+E_{110}+F_{111}+G_{100}+H_{101}$ $A_{010}+B_{011}+C_{000}-D_{001}+E_{110}+F_{111}+G_{100}+H_{101}$ $A_{010}+B_{011}+C_{000}+D_{001}+E_{110}-F_{111}+G_{100}+H_{101}$ $A_{010}+B_{011}+C_{000}+D_{001}+E_{110}+F_{111}+G_{100}-H_{101}$ $A_{010}-B_{011}+C_{000}-D_{001}+E_{110}+F_{111}+G_{100}+H_{101}$ $A_{010}-B_{011}+C_{000}+D_{001}+E_{110}-F_{111}+G_{100}+H_{101}$ $A_{010}-B_{011}+C_{000}+D_{001}+E_{110}+F_{111}+G_{100}-H_{101}$ $A_{010}+B_{011}+C_{000}+D_{001}+E_{110}+F_{111}+G_{100}+H_{101}$ $A_{010}+B_{011}+C_{000}-D_{001}+E_{110}-F_{111}+G_{100}+H_{101}$ $A_{010}+B_{011}+C_{000}-D_{001}+E_{110}+F_{111}+G_{100}-H_{101}$ $A_{010}+B_{011}+C_{000}+D_{001}+E_{110}-F_{111}+G_{100}-H_{101}$ $A_{010}-B_{011}+C_{000}-D_{001}+E_{110}-F_{111}+G_{100}+H_{101}$ $A_{010}-B_{011}+C_{000}-D_{001}+E_{110}+F_{111}+G_{100}-H_{101}$ $A_{010}+B_{011}+C_{000}-D_{001}+E_{110}-F_{111}+G_{100}-H_{101}$ $A_{010}-B_{011}+C_{000}+D_{001}+E_{110}-F_{111}+G_{100}-H_{101}$ $A_{010}-B_{011}+C_{000}-D_{001}+E_{110}-F_{111}+G_{100}-H_{101}$ $-A_{010}+B_{011}+C_{000}+D_{001}+E_{110}+F_{111}+G_{100}+H_{101}$ $A_{010}+B_{011}+C_{000}+D_{001}-E_{110}+F_{111}+G_{100}+H_{101}$ $-A_{010}-B_{011}+C_{000}+D_{001}+E_{110}+F_{111}+G_{100}+H_{101}$ $-A_{010}+B_{011}+C_{000}+D_{001}-E_{110}+F_{111}+G_{100}+H_{101}$ $-A_{010}+B_{011}+C_{000}+D_{001}+E_{110}-F_{111}+G_{100}+H_{101}$ $A_{010}-B_{011}+C_{000}+D_{001}-E_{110}+F_{111}+G_{100}+H_{101}$ $A_{010}+B_{011}+C_{000}+D_{001}-E_{110}-F_{111}+G_{100}+H_{101}$ $-A_{010}-B_{011}+C_{000}+D_{001}-E_{110}+F_{111}+G_{100}+H_{101}$ $-A_{010}+B_{011}+C_{000}+D_{001}-E_{110}-F_{111}+G_{100}+H_{101}$ $A_{010}-B_{011}+C_{000}+D_{001}-E_{110}-F_{111}+G_{100}+H_{101}$ $-A_{010}-B_{011}+C_{000}+D_{001}+E_{110}-F_{111}+G_{100}+H_{101}$ $-A_{010}-B_{011}+C_{000}+D_{001}-E_{110}-F_{111}+G_{100}+H_{101}$ $A_{010}+B_{011}+C_{000}+D_{001}+E_{110}+F_{111}-G_{100}+H_{101}$ $A_{010}+B_{011}+C_{000}+D_{001}-E_{110}+F_{111}-G_{100}+H_{101}$ $A_{010}+B_{011}+C_{000}+D_{001}-E_{110}+F_{111}+G_{100}-H_{101}$ $A_{010}+B_{011}+C_{000}+D_{001}+E_{110}-F_{111}-G_{100}+H_{101}$ $A_{010}+B_{011}+C_{000}+D_{001}+E_{110}-F_{111}+G_{100}-H_{101}$ $A_{010}+B_{011}+C_{000}+D_{001}-E_{110}-F_{111}-G_{100}+H_{101}$ $A_{010}+B_{011}+C_{000}+D_{001}-E_{110}+F_{111}-G_{100}-H_{101}$ $A_{010}+B_{011}+C_{000}+D_{001}+E_{110}-F_{111}-G_{100}-H_{101}$ $A_{010}+B_{011}+C_{000}+D_{001}-E_{110}-F_{111}+G_{100}-H_{101}$ $A_{010}+B_{011}+C_{000}+D_{001}-E_{110}-F_{111}-G_{100}-H_{101}$ $A_{011}+B_{010}+C_{001}+D_{000}-E_{111}-F_{110}-G_{101}-H_{100}$ $A_{011}+B_{010}+C_{001}+D_{000}+E_{111}+F_{110}+G_{101}+H_{100}$ $-A_{011}+B_{010}+C_{001}+D_{000}+E_{111}+F_{110}+G_{101}+H_{100}$ $A_{011}+B_{010}-C_{001}+D_{000}+E_{111}+F_{110}+G_{101}+H_{100}$ $A_{011}+B_{010}+C_{001}+D_{000}-E_{111}+F_{110}+G_{101}+H_{100}$ $A_{011}+B_{010}+C_{001}+D_{000}+E_{111}+F_{110}-G_{101}+H_{100}$ $-A_{011}+B_{010}-C_{001}+D_{000}+E_{111}+F_{110}+G_{101}+H_{100}$ $\sigma_{x} \otimes P_{1} \otimes P_{1}+\sigma_{x} \otimes P_{2} \otimes P_{1}+I \sigma_{y} \otimes P_{1} \otimes P_{2}-\sigma_{x} \otimes P_{2} \otimes P_{2}$ $\sigma_{x} \otimes P_{1} \otimes P_{1}-\sigma_{x} \otimes P_{2} \otimes P_{1}+\sigma_{x} \otimes P_{1} \otimes P_{2}+I \sigma_{y} \otimes P_{2} \otimes P_{2}$ $\sigma_{x} \otimes P_{1} \otimes P_{1}-\sigma_{x} \otimes P_{2} \otimes P_{1}+\sigma_{x} \otimes P_{1} \otimes P_{2}-I \sigma_{y} \otimes P_{2} \otimes P_{2}$ $\sigma_{x} \otimes P_{1} \otimes P_{1}-\sigma_{x} \otimes P_{2} \otimes P_{1}+\sigma_{x} \otimes P_{1} \otimes P_{2}-I \sigma_{y} \otimes P_{2} \otimes P_{2}$ $\sigma_{x} \otimes P_{1} \otimes P_{1}-I \sigma_{y} \otimes P_{2} \otimes P_{1}+\sigma_{x} \otimes P_{1} \otimes P_{2}-\sigma_{x} \otimes P_{2} \otimes P_{2}$ $\sigma_{x} \otimes P_{1} \otimes P_{1}-\sigma_{x} \otimes P_{2} \otimes P_{1}+\sigma_{x} \otimes P_{1} \otimes P_{2}-\sigma_{x} \otimes P_{2} \otimes P_{2}$ $\sigma_{x} \otimes P_{1} \otimes P_{1}+\sigma_{x} \otimes P_{2} \otimes P_{1}-I \sigma_{y} \otimes P_{1} \otimes P_{2}+\sigma_{x} \otimes P_{2} \otimes P_{2}$ $\sigma_{x} \otimes P_{1} \otimes P_{1}+\sigma_{x} \otimes P_{2} \otimes P_{1}+I \sigma_{y} \otimes P_{1} \otimes P_{2}+\sigma_{x} \otimes P_{2} \otimes P_{2}$ $\sigma_{x} \otimes P_{1} \otimes P_{1}+\sigma_{x} \otimes P_{2} \otimes P_{1}+I \sigma_{y} \otimes P_{1} \otimes P_{2}+I \sigma_{y} \otimes P_{2} \otimes P_{2}$ $\sigma_{x} \otimes P_{1} \otimes P_{1}+\sigma_{x} \otimes P_{2} \otimes P_{1}+I \sigma_{y} \otimes P_{1} \otimes P_{2}-I \sigma_{y} \otimes P_{2} \otimes P_{2}$ $\sigma_{x} \otimes P_{1} \otimes P_{1}+\sigma_{x} \otimes P_{2} \otimes P_{1}-I \sigma_{y} \otimes P_{1} \otimes P_{2}+I \sigma_{y} \otimes P_{2} \otimes P_{2}$ $\sigma_{x} \otimes P_{1} \otimes P_{1}+\sigma_{x} \otimes P_{2} \otimes P_{1}-I \sigma_{y} \otimes P_{1} \otimes P_{2}-I \sigma_{y} \otimes P_{2} \otimes P_{2}$ $\sigma_{x} \otimes P_{1} \otimes P_{1}+\sigma_{x} \otimes P_{2} \otimes P_{1}-\sigma_{x} \otimes P_{1} \otimes P_{2}+I \sigma_{y} \otimes P_{2} \otimes P_{2}$ $\sigma_{x} \otimes P_{1} \otimes P_{1}+\sigma_{x} \otimes P_{2} \otimes P_{1}+I \sigma_{y} \otimes P_{1} \otimes P_{2}-\sigma_{x} \otimes P_{2} \otimes P_{2}$ $\sigma_{x} \otimes P_{1} \otimes P_{1}+\sigma_{x} \otimes P_{2} \otimes P_{1}-I \sigma_{y} \otimes P_{1} \otimes P_{2}-\sigma_{x} \otimes P_{2} \otimes P_{2}$ $\sigma_{x} \otimes P_{1} \otimes P_{1}+\sigma_{x} \otimes P_{2} \otimes P_{1}-\sigma_{x} \otimes P_{1} \otimes P_{2}-\sigma_{x} \otimes P_{2} \otimes P_{2}$ $\sigma_{z} \otimes F_{1} \otimes P_{1}+I_{2} \otimes F_{2} \otimes P_{1}+I_{2} \otimes F_{1} \otimes P_{2}+I_{2} \otimes P_{2} \otimes P_{2}$ $I_{2} \otimes F_{1} \otimes P_{1}+\sigma_{z} \otimes F_{2} \otimes P_{1}+I_{2} \otimes F_{1} \otimes P_{2}+I_{2} \otimes P_{2} \otimes P_{2}$ $I_{2} \otimes F_{1} \otimes P_{1}+I_{2} \otimes F_{2} \otimes P_{1}+\sigma_{z} \otimes F_{1} \otimes P_{2}+I_{2} \otimes P_{2} \otimes P_{2}$ $\sigma_{z} \otimes F_{1} \otimes P_{1}+I_{2} \otimes F_{2} \otimes P_{1}+I_{2} \otimes F_{1} \otimes P_{2}+\sigma_{z} \otimes P_{2} \otimes P_{2}$ $\sigma_{z} \otimes F_{1} \otimes P_{1}+\sigma_{z} \otimes F_{2} \otimes P_{1}+I_{2} \otimes F_{1} \otimes P_{2}+I_{2} \otimes P_{2} \otimes P_{2}$ $\sigma_{z} \otimes F_{1} \otimes P_{1}+I_{2} \otimes F_{2} \otimes P_{1}+\sigma_{z} \otimes F_{1} \otimes P_{2}+I_{2} \otimes P_{2} \otimes P_{2}$ $\sigma_{z} \otimes F_{1} \otimes P_{1}+I_{2} \otimes F_{2} \otimes P_{1}+I_{2} \otimes F_{1} \otimes P_{2}+\sigma_{z} \otimes P_{2} \otimes P_{2}$ $I_{2} \otimes F_{1} \otimes P_{1}+I_{2} \otimes F_{2} \otimes P_{1}+I_{2} \otimes F_{1} \otimes P_{2}+I_{2} \otimes P_{2} \otimes P_{2}$ $I_{2} \otimes F_{1} \otimes P_{1}+\sigma_{z} \otimes F_{2} \otimes P_{1}+\sigma_{z} \otimes F_{1} \otimes P_{2}+I_{2} \otimes P_{2} \otimes P_{2}$ $I_{2} \otimes F_{1} \otimes P_{1}+\sigma_{z} \otimes F_{2} \otimes P_{1}+I_{2} \otimes F_{1} \otimes P_{2}+\sigma_{z} \otimes P_{2} \otimes P_{2}$ $I_{2} \otimes F_{1} \otimes P_{1}+I_{2} \otimes F_{2} \otimes P_{1}+\sigma_{z} \otimes F_{1} \otimes P_{2}+\sigma_{z} \otimes P_{2} \otimes P_{2}$ $\sigma_{z} \otimes F_{1} \otimes P_{1}+\sigma_{z} \otimes F_{2} \otimes P_{1}+I_{2} \otimes F_{1} \otimes P_{2}+I_{2} \otimes P_{2} \otimes P_{2}$ $\sigma_{z} \otimes F_{1} \otimes P_{1}+\sigma_{z} \otimes F_{2} \otimes P_{1}+I_{2} \otimes F_{1} \otimes P_{2}+\sigma_{z} \otimes P_{2} \otimes P_{2}$ $I_{2} \otimes F_{1} \otimes P_{1}+\sigma_{z} \otimes F_{2} \otimes P_{1}+\sigma_{z} \otimes F_{1} \otimes P_{2}+\sigma_{z} \otimes P_{2} \otimes P_{2}$ $\sigma_{z} \otimes F_{1} \otimes P_{1}+I_{2} \otimes F_{2} \otimes P_{1}+\sigma_{z} \otimes F_{1} \otimes P_{2}+\sigma_{z} \otimes P_{2} \otimes P_{2}$ $\sigma_{z} \otimes F_{1} \otimes P_{1}+\sigma_{z} \otimes F_{2} \otimes P_{1}+\sigma_{z} \otimes P_{2} \otimes P_{2}+\sigma_{z} \otimes P_{2} \otimes P_{2}$ $\sigma_{z} \otimes F_{1} \otimes P_{1}+I_{2} \otimes F_{2} \otimes P_{1}+I_{2} \otimes F_{1} \otimes P_{2}+I_{2} \otimes P_{2} \otimes P_{2}$ $I_{2} \otimes F_{1} \otimes P_{1}+I_{2} \otimes F_{2} \otimes P_{1}-\sigma_{z} \otimes F_{1} \otimes P_{2}+I_{2} \otimes P_{2} \otimes P_{2}$ $-I_{2} \otimes F_{1} \otimes P_{1}+I_{2} \otimes F_{2} \otimes P_{1}+I_{2} \otimes F_{1} \otimes P_{2}+I_{2} \otimes P_{2} \otimes P_{2}$ $-\sigma_{z} \otimes F_{1} \otimes P_{1}+I_{2} \otimes F_{2} \otimes P_{1}-\sigma_{z} \otimes F_{1} \otimes P_{2}+I_{2} \otimes P_{2} \otimes P_{2}$ $-\sigma_{z} \otimes F_{1} \otimes P_{1}+I_{2} \otimes F_{2} \otimes P_{1}+\sigma_{z} \otimes F_{1} \otimes P_{2}+I_{2} \otimes P_{2} \otimes P_{2}$ $-\sigma_{z} \otimes F_{1} \otimes P_{1}+I_{2} \otimes F_{2} \otimes P_{1}-\sigma_{z} \otimes F_{1} \otimes P_{2}+I_{2} \otimes P_{2} \otimes P_{2}$ $I_{2} \otimes F_{1} \otimes P_{1}+I_{2} \otimes F_{2} \otimes P_{1}-I_{2} \otimes F_{1} \otimes P_{2}+I_{2} \otimes P_{2} \otimes P_{2}$ $-I_{2} \otimes F_{1} \otimes P_{1}+I_{2} \otimes F_{2} \otimes P_{1}-\sigma_{z} \otimes F_{1} \otimes P_{2}+I_{2} \otimes P_{2} \otimes P_{2}$ $-\sigma_{z} \otimes F_{1} \otimes P_{1}+I_{2} \otimes F_{2} \otimes P_{1}-I_{2} \otimes F_{1} \otimes P_{2}+I_{2} \otimes P_{2} \otimes P_{2}$ $\sigma_{z} \otimes F_{1} \otimes P_{1}+I_{2} \otimes F_{2} \otimes P_{1}-I_{2} \otimes F_{1} \otimes P_{2}+I_{2} \otimes P_{2} \otimes P_{2}$ $-I_{2} \otimes F_{1} \otimes P_{1}+I_{2} \otimes F_{2} \otimes P_{1}+\sigma_{z} \otimes F_{1} \otimes P_{2}+I_{2} \otimes P_{2} \otimes P_{2}$ $-I_{2} \otimes F_{1} \otimes P_{1}+I_{2} \otimes F_{2} \otimes P_{1}-I_{2} \otimes F_{1} \otimes P_{2}+I_{2} \otimes P_{2} \otimes P_{2}$ $I_{2} \otimes F_{1} \otimes P_{1}+I_{2} \otimes F_{2} \otimes P_{1}+I_{2} \otimes F_{1} \otimes P_{2}-\sigma_{z} \otimes P_{2} \otimes P_{2}$ $I_{2} \otimes F_{1} \otimes P_{1}+I_{2} \otimes F_{2} \otimes P_{1}-\sigma_{z} \otimes F_{1} \otimes P_{2}-\sigma_{z} \otimes P_{2} \otimes P_{2}$ $I_{2} \otimes F_{1} \otimes P_{1}+I_{2} \otimes F_{2} \otimes P_{1}-\sigma_{z} \otimes F_{1} \otimes P_{2}+\sigma_{z} \otimes P_{2} \otimes P_{2}$ $I_{2} \otimes F_{1} \otimes P_{1}+I_{2} \otimes F_{2} \otimes P_{1}+\sigma_{z} \otimes F_{1} \otimes P_{2}-\sigma_{z} \otimes P_{2} \otimes P_{2}$ $I_{2} \otimes F_{1} \otimes P_{1}+I_{2} \otimes F_{2} \otimes P_{1}+\sigma_{z} \otimes F_{1} \otimes P_{2}+\sigma_{z} \otimes P_{2} \otimes P_{2}$ $I_{2} \otimes F_{1} \otimes P_{1}+I_{2} \otimes F_{2} \otimes P_{1}-I_{2} \otimes F_{1} \otimes P_{2}-\sigma_{z} \otimes P_{2} \otimes P_{2}$ $I_{2} \otimes F_{1} \otimes P_{1}+I_{2} \otimes F_{2} \otimes P_{1}-\sigma_{z} \otimes F_{1} \otimes P_{2}-I_{2} \otimes P_{2} \otimes P_{2}$ $I_{2} \otimes F_{1} \otimes P_{1}+I_{2} \otimes F_{2} \otimes P_{1}+\sigma_{z} \otimes F_{1} \otimes P_{2}-I_{2} \otimes P_{2} \otimes P_{2}$ $I_{2} \otimes F_{1} \otimes P_{1}+I_{2} \otimes F_{2} \otimes P_{1}-I_{2} \otimes F_{1} \otimes P_{2}+\sigma_{z} \otimes P_{2} \otimes P_{2}$ $I_{2} \otimes F_{1} \otimes P_{1}+I_{2} \otimes F_{2} \otimes P_{1}-I_{2} \otimes F_{1} \otimes P_{2}-I_{2} \otimes P_{2} \otimes P_{2}$ $\sigma_{x} \otimes \sigma_{x} \otimes P_{1}-\sigma_{x} \otimes \sigma_{x} \otimes P_{2}$ $\sigma_{x} \otimes \sigma_{x} \otimes I_{2}$ $I \sigma_{y} \otimes F_{1} \otimes P_{1}+\sigma_{x} \otimes F_{2} \otimes P_{1}+\sigma_{x} \otimes \sigma_{x} \otimes P_{2}$ $\sigma_{x} \otimes F_{1} \otimes P_{1}+I \sigma_{y} \otimes F_{2} \otimes P_{1}-I \sigma_{y} \otimes F_{1} \otimes P_{2}+\sigma_{x} \otimes F_{2} \otimes P_{2}$ $\sigma_{x} \otimes \sigma_{x} \otimes P_{1}+I \sigma_{y} \otimes F_{1} \otimes P_{2}+\sigma_{x} \otimes F_{2} \otimes P_{2}$ $\sigma_{x} \otimes \sigma_{x} \otimes P_{1}+\sigma_{x} \otimes F_{1} \otimes P_{2}+I \sigma_{y} \otimes F_{2} \otimes P_{2}$

$I \sigma_{y} \otimes \sigma_{x} \otimes P_{1}+\sigma_{x} \otimes F_{1} \otimes P_{2}+\sigma_{x} \otimes F_{2} \otimes P_{2}$ 







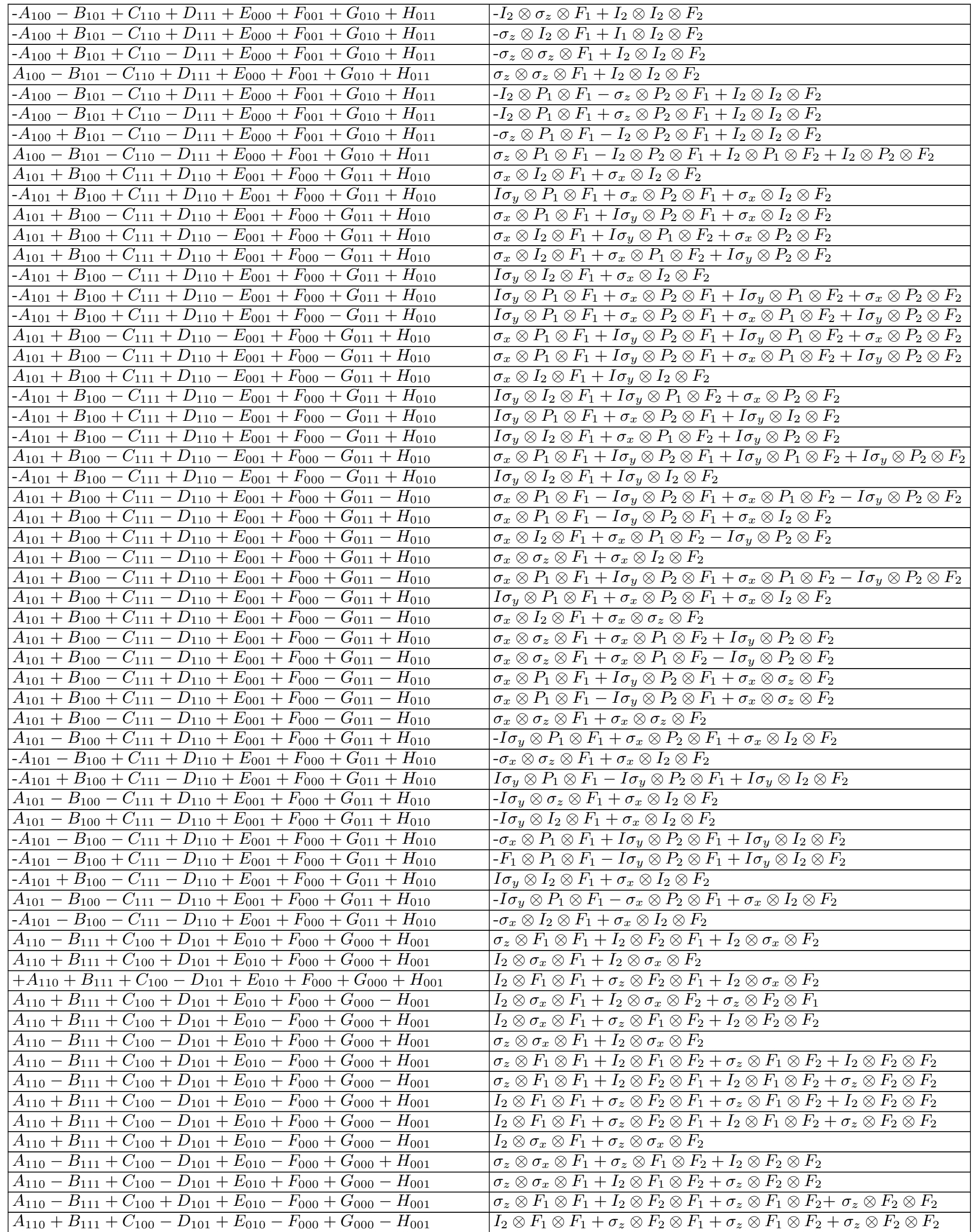


$A_{110}-B_{111}+C_{100}-D_{101}+E_{010}-F_{000}+G_{000}-H_{001}$ $-A_{110}+B_{111}+C_{100}+D_{101}+E_{010}+F_{000}+G_{000}+H_{001}$ $A_{110}+B_{111}+C_{100}+D_{101}-E_{010}+F_{000}+G_{000}+H_{001}$ $-A_{110}-B_{111}+C_{100}+D_{101}+E_{010}+F_{000}+G_{000}+H_{001}$ $-A_{110}+B_{111}+C_{100}+D_{101}-E_{010}+F_{000}+G_{000}+H_{001}$ $-A_{110}+B_{111}+C_{100}+D_{101}+E_{010}-F_{000}+G_{000}+H_{001}$ $A_{110}-B_{111}+C_{100}+D_{101}-E_{010}+F_{000}+G_{000}+H_{001}$ $A_{110}+B_{111}+C_{100}+D_{101}-E_{010}-F_{000}+G_{000}+H_{001}$ $-A_{110}-B_{111}+C_{100}+D_{101}-E_{010}+F_{000}+G_{000}+H_{001}$ $-A_{110}+B_{111}+C_{100}+D_{101}-E_{010}-F_{000}+G_{000}+H_{001}$ $-A_{110}-B_{111}+C_{100}+D_{101}+E_{010}-F_{000}+G_{000}+H_{001}$ $A_{110}-B_{111}+C_{100}+D_{101}-E_{010}-F_{000}+G_{000}+H_{001}$ $-A_{110}-B_{111}+C_{100}+D_{101}-E_{010}-F_{000}+G_{000}+H_{001}$ $A_{110}+B_{111}-C_{100}+D_{101}+E_{010}+F_{000}+G_{000}+H_{001}$ $-A_{110}+B_{111}-C_{100}+D_{101}+E_{010}+F_{000}+G_{000}+H_{001}$ $-A_{110}+B_{111}+C_{100}-D_{101}+E_{010}+F_{000}+G_{000}+H_{001}$ $A_{110}-B_{111}-C_{100}+D_{101}+E_{010}+F_{000}+G_{000}+H_{001}$ $A_{110}+B_{111}-C_{100}-D_{101}+E_{010}+F_{000}+G_{000}+H_{001}$ $-A_{110}-B_{111}-C_{100}+D_{101}+E_{010}+F_{000}+G_{000}+H_{001}$ $-A_{110}-B_{111}+C_{100}-D_{101}+E_{010}+F_{000}+G_{000}+H_{001}$ $-A_{110}+B_{111}-C_{100}-D_{101}+E_{010}+F_{000}+G_{000}+H_{001}$ $A_{110}-B_{111}-C_{100}-D_{101}+E_{010}+F_{000}+G_{000}+H_{001}$ $-A_{110}-B_{111}-C_{100}-D_{101}+E_{010}+F_{000}+G_{000}+H_{001}$ $-A_{111}+B_{110}+C_{101}+D_{100}+E_{011}+F_{010}+G_{001}+H_{000}$ $A_{111}+B_{110}-C_{101}+D_{100}+E_{011}+F_{010}+G_{001}+H_{000}$ $A_{111}+B_{110}+C_{101}+D_{100}-E_{011}+F_{010}+G_{001}+H_{000}$ $A_{111}+B_{110}+C_{101}+D_{100}+E_{011}+F_{010}-G_{001}+H_{000}$ $-A_{111}+B_{110}-C_{101}+D_{100}+E_{011}+F_{010}+G_{001}+H_{000}$ $-A_{111}+B_{110}+C_{101}+D_{100}-E_{011}+F_{010}+G_{001}+H_{000}$ $-A_{111}+B_{110}+C_{101}+D_{100}+E_{011}+F_{010}-G_{001}+H_{000}$ $A_{111}+B_{110}-C_{101}+D_{100}-E_{011}+F_{010}+G_{001}+H_{000}$ $A_{111}+B_{110}-C_{101}+D_{100}+E_{011}+F_{010}-G_{001}+H_{000}$ $A_{111}+B_{110}+C_{101}+D_{100}-E_{011}+F_{010}-G_{001}+H_{000}$ $-A_{111}+B_{110}-C_{101}+D_{100}-E_{011}+F_{010}+G_{001}+H_{000}$ $-A_{111}+B_{110}+C_{101}+D_{100}-E_{011}+F_{010}-G_{001}+H_{000}$ $-A_{111}+B_{110}-C_{101}+D_{100}+E_{011}+F_{010}-G_{001}+H_{000}$ $A_{111}+B_{110}-C_{101}+D_{100}-E_{011}+F_{010}-G_{001}+H_{000}$ $-A_{111}+B_{110}-C_{101}+D_{100}-E_{011}+F_{010}-G_{001}+H_{000}$ $A_{111}+B_{110}+C_{101}+D_{100}+E_{011}+F_{010}+G_{001}+H_{000}$ $A_{111}-B_{110}+C_{101}+D_{100}+E_{011}+F_{010}+G_{001}+H_{000}$ $A_{111}+B_{110}+C_{101}+D_{100}+E_{011}-F_{010}+G_{001}+H_{000}$ $-A_{111}-B_{110}+C_{101}+D_{100}+E_{011}+F_{010}+G_{001}+H_{000}$ $-A_{111}+B_{110}+C_{101}+D_{100}+E_{011}-F_{010}+G_{001}+H_{000}$ $A_{111}-B_{110}+C_{101}+D_{100}-E_{011}+F_{010}+G_{001}+H_{000}$ $A_{111}-B_{110}+C_{101}+D_{100}+E_{011}-F_{010}+G_{001}+H_{000}$ $A_{111}+B_{110}+C_{101}+D_{100}-E_{011}-F_{010}+G_{001}+H_{000}$ $-A_{111}-B_{110}+C_{101}+D_{100}-E_{011}+F_{010}+G_{001}+H_{000}$ $-A_{111}+B_{110}+C_{101}+D_{100}-E_{011}-F_{010}+G_{001}+H_{000}$ $-A_{111}-B_{110}+C_{101}+D_{100}-E_{011}+F_{010}+G_{001}+H_{000}$ $A_{111}-B_{110}+C_{101}+D_{100}-E_{011}-F_{010}+G_{001}+H_{000}$ $-A_{111}-B_{110}+C_{101}+D_{100}-E_{011}-F_{010}+G_{001}+H_{000}$ $A_{111}+B_{110}+C_{101}-D_{100}+E_{011}+F_{010}+G_{001}+H_{000}$ $-A_{111}+B_{110}+C_{101}-D_{100}+E_{011}+F_{010}+G_{001}+H_{000}$ $A_{111}-B_{110}-C_{101}+D_{100}+E_{011}+F_{010}+G_{001}+H_{000}$ $A_{111}-B_{110}+C_{101}-D_{100}+E_{011}+F_{010}+G_{001}+H_{000}$ $A_{111}+B_{110}-C_{101}-D_{100}+E_{011}+F_{010}+G_{001}+H_{000}$ $-A_{111}-B_{110}-C_{101}+D_{100}+E_{011}+F_{010}+G_{001}+H_{000}$ $-A_{111}-B_{110}+C_{101}-D_{100}+E_{011}+F_{010}+G_{001}+H_{000}$ $-A_{111}+B_{110}-C_{101}-D_{100}+E_{011}+F_{010}+G_{001}+H_{000}$ $A_{111}-B_{110}-C_{101}-D_{100}+E_{011}+F_{010}+G_{001}+H_{000}$ $-A_{111}-B_{110}-C_{101}-D_{100}+E_{011}+F_{010}+G_{001}+H_{000}$ $\sigma_{z} \otimes \sigma_{x} \otimes F_{1}+\sigma_{z} \otimes \sigma_{x} \otimes F_{2}$

$-\sigma_{z} \otimes F_{1} \otimes F_{1}+I_{2} \otimes F_{2} \otimes F_{1}+I_{2} \otimes \sigma_{x} \otimes F_{2}$

$I_{2} \otimes \sigma_{x} \otimes F_{1}-\sigma_{z} \otimes F_{1} \otimes F_{2}+I_{2} \otimes F_{2} \otimes F_{2}$

$I_{2} \otimes I \sigma_{y} \otimes F_{1}+I_{2} \otimes \sigma_{x} \otimes F_{2}$

$-\sigma_{z} \otimes F_{1} \otimes F_{1}+I_{2} \otimes F_{2} \otimes F_{1}-\sigma_{z} \otimes F_{1} \otimes F_{2}+I_{2} \otimes F_{2} \otimes F_{2}$

$-\sigma_{z} \otimes F_{1} \otimes F_{1}+I_{2} \otimes F_{2} \otimes F_{1}+\sigma_{z} \otimes F_{1} \otimes F_{2}+I_{2} \otimes F_{2} \otimes F_{2}$

$\sigma_{z} \otimes F_{1} \otimes F_{1}+I_{2} \otimes F_{2} \otimes F_{1}-\sigma_{z} \otimes F_{1} \otimes F_{2}+I_{2} \otimes F_{2} \otimes F_{2}$

$I_{2} \otimes \sigma_{x} \otimes F_{1}+I_{2} \otimes I \sigma_{y} \otimes F_{2}$

$\sigma_{z} \otimes F_{1} \otimes F_{1}+I_{2} \otimes F_{2} \otimes F_{1}-\sigma_{z} \otimes F_{1} \otimes F_{2}+I_{2} \otimes F_{2} \otimes F_{2}$

$-\sigma_{z} \otimes F_{1} \otimes F_{1}+I_{2} \otimes F_{2} \otimes F_{1}+I_{2} \otimes I \sigma_{y} \otimes F_{2}$

$I_{2} \otimes I \sigma_{y} \otimes F_{1}+\sigma_{z} \otimes F_{1} \otimes F_{2}+I_{2} \otimes F_{2} \otimes F_{2}$

$\sigma_{z} \otimes F_{1} \otimes F_{1}+I_{2} \otimes F_{2} \otimes F_{1}+I_{2} \otimes I \sigma_{y} \otimes F_{2}$

$I_{2} \otimes I \sigma_{y} \otimes F_{1}+I_{2} \otimes I \sigma_{y} \otimes F_{2}$

$I_{2} \otimes F_{1} \otimes F_{1}-\sigma_{z} \otimes F_{2} \otimes F_{1}+I_{2} \otimes \sigma_{x} \otimes F_{2}$

$-\sigma_{z} \otimes F_{1} \otimes F_{1}-\sigma_{z} \otimes F_{2} \otimes F_{1}+I_{2} \otimes \sigma_{x} \otimes F_{2}$

$\sigma_{z} \otimes I \sigma_{y} \otimes F_{1}+I_{2} \otimes \sigma_{x} \otimes F_{2}$

$-\sigma_{z} \otimes I \sigma_{y} \otimes F_{1}+I_{2} \otimes \sigma_{x} \otimes F_{2}$

$I_{2} \otimes I \sigma_{y} \otimes F_{1}+I_{2} \otimes \sigma_{x} \otimes F_{2}$

$I_{2} \otimes F_{1} \otimes F_{1}-\sigma_{z} \otimes F_{2} \otimes F_{1}+I_{2} \otimes \sigma_{x} \otimes F_{2}$

$-I_{2} \otimes F_{1} \otimes F_{1}+\sigma_{z} \otimes F_{2} \otimes F_{1}+I_{2} \otimes \sigma_{x} \otimes F_{2}$

$-\sigma_{z} \otimes F_{1} \otimes F_{1}-I_{2} \otimes F_{2} \otimes F_{1}+I_{2} \otimes \sigma_{x} \otimes F_{2}$

$\sigma_{z} \otimes F_{1} \otimes F_{1}-I_{2} \otimes F_{2} \otimes F_{1}+I_{2} \otimes \sigma_{x} \otimes F_{2}$

$-I_{2} \otimes \sigma_{x} \otimes F_{1}+I_{2} \otimes \sigma_{x} \otimes F_{2}$

$I \sigma_{y} \otimes F_{1} \otimes F_{1}+\sigma_{x} \otimes F_{2} \otimes F_{1}+\sigma_{x} \otimes \sigma_{x} \otimes F_{2}$

$\sigma_{x} \otimes F_{1} \otimes F_{1}+I \sigma_{y} \otimes F_{2} \otimes F_{1}+\sigma_{x} \otimes \sigma_{x} \otimes F_{2}$

$\sigma_{x} \otimes \sigma_{x} \otimes F_{1}+I \sigma_{y} \otimes F_{1} \otimes F_{2}+\sigma_{x} \otimes F_{2} \otimes F_{2}$

$\sigma_{x} \otimes \sigma_{x} \otimes F_{1}+\sigma_{x} \otimes F_{1} \otimes F_{2}+I \sigma_{y} \otimes F_{2} \otimes F_{2}$

$I \sigma_{y} \otimes \sigma_{x} \otimes F_{1}+\sigma_{x} \otimes \sigma_{x} \otimes F_{2}$

$I \sigma_{y} \otimes F_{1} \otimes F_{1}+\sigma_{x} \otimes F_{2} \otimes F_{1}+I \sigma_{y} \otimes F_{1} \otimes F_{2}+\sigma_{x} \otimes F_{2} \otimes F_{2}$ $I \sigma_{y} \otimes F_{1} \otimes F_{1}+\sigma_{x} \otimes F_{2} \otimes F_{1}+I \sigma_{y} \otimes F_{1} \otimes F_{2}+\sigma_{x} \otimes F_{2} \otimes F_{2}$ $\sigma_{x} \otimes F_{1} \otimes F_{1}+I \sigma_{y} \otimes F_{2} \otimes F_{1}+I \sigma_{y} \otimes F_{1} \otimes F_{2}+\sigma_{x} \otimes F_{2} \otimes F_{2}$ $\sigma_{x} \otimes F_{1} \otimes F_{1}+I \sigma_{y} \otimes F_{2} \otimes F_{1}+\sigma_{x} \otimes F_{1} \otimes F_{2}+I \sigma_{y} \otimes F_{2} \otimes F_{2}$ $\sigma_{x} \otimes \sigma_{x} \otimes F_{1}+\sigma_{x} \otimes F_{2} \otimes F_{1}+I \sigma_{y} \otimes \sigma_{x} \otimes F_{2}$

$I \sigma_{y} \otimes \sigma_{x} \otimes F_{1}+I \sigma_{y} \otimes F_{1} \otimes F_{2}+\sigma_{x} \otimes F_{2} \otimes F_{2}$

$I \sigma_{y} \otimes F_{1} \otimes F_{1}+\sigma_{x} \otimes F_{2} \otimes F_{1}+I \sigma_{y} \otimes \sigma_{x} \otimes F_{2}$

$I \sigma_{y} \otimes \sigma_{x} \otimes F_{1}+\sigma_{x} \otimes F_{1} \otimes F_{2}+I \sigma_{y} \otimes F_{2} \otimes F_{2}$

$\sigma_{x} \otimes F_{1} \otimes F_{1}+I \sigma_{y} \otimes F_{2} \otimes F_{1}+I \sigma_{y} \otimes \sigma_{x} \otimes F_{2}$

$I \sigma_{y} \otimes \sigma_{x} \otimes F_{1}+I \sigma_{y} \otimes \sigma_{x} \otimes F_{2}$

$\sigma_{x} \otimes \sigma_{x} \otimes \sigma_{x}$

$I \sigma_{y} \otimes F_{1} \otimes F_{1}+\sigma_{x} \otimes F_{2} \otimes F_{1}+\sigma_{x} \otimes \sigma_{x} \otimes F_{2}$

$\sigma_{x} \otimes \sigma_{x} \otimes F_{1}-I \sigma_{y} \otimes F_{1} \otimes F_{2}+\sigma_{x} \otimes F_{2} \otimes F_{2}$

$\sigma_{x} \otimes I \sigma_{y} \otimes F_{1}+\sigma_{x} \otimes \sigma_{x} \otimes F_{2}$

$I \sigma_{y} \otimes F_{1} \otimes F_{1}+\sigma_{x} \otimes F_{2} \otimes F_{1}-I \sigma_{y} \otimes F_{1} \otimes F_{2}+\sigma_{x} \otimes F_{2} \otimes F_{2}$

$-I \sigma_{y} \otimes F_{1} \otimes F_{1}+\sigma_{x} \otimes F_{2} \otimes F_{1}+I \sigma_{y} \otimes F_{1} \otimes F_{2}+\sigma_{x} \otimes F_{2} \otimes F_{2}$

$-I \sigma_{y} \otimes F_{1} \otimes F_{1}+\sigma_{x} \otimes F_{2} \otimes F_{1}-I \sigma_{y} \otimes F_{1} \otimes F_{2}+\sigma_{x} \otimes F_{2} \otimes F_{2}$

$\sigma_{x} \otimes \sigma_{x} \otimes F_{1}+\sigma_{x} \otimes I \sigma_{y} \otimes F_{2}$

$\sigma_{x} \otimes I \sigma_{y} \otimes F_{1}+I \sigma_{y} \otimes F_{1} \otimes F_{2}+\sigma_{x} \otimes F_{2} \otimes F_{2}$

$I \sigma_{y} \otimes F_{1} \otimes F_{1}+\sigma_{x} \otimes F_{2} \otimes F_{1}+\sigma_{x} \otimes \sigma_{x} \otimes F_{2}$

$\sigma_{x} \otimes I \sigma_{y} \otimes F_{1}+I \sigma_{y} \otimes F_{1} \otimes F_{2}+\sigma_{x} \otimes F_{2} \otimes F_{2}$

$-I \sigma_{y} \otimes F_{1} \otimes F_{1}+\sigma_{x} \otimes F_{2} \otimes F_{1}+\sigma_{x} \otimes I \sigma_{y} \otimes F_{2}$

$\sigma_{x} \otimes I \sigma_{y} \otimes \sigma_{x}$

$\sigma_{x} \otimes F_{1} \otimes F_{1}-I \sigma_{y} \otimes F_{2} \otimes F_{1}+\sigma_{x} \otimes \sigma_{x} \otimes F_{2}$

$-I \sigma_{y} \otimes I \sigma_{y} \otimes F_{1}+\sigma_{x} \otimes \sigma_{x} \otimes F_{2}$

$I \sigma_{y} \otimes I \sigma_{y} \otimes F_{1}+\sigma_{x} \otimes \sigma_{x} \otimes F_{2}$

$-I \sigma_{y} \otimes \sigma_{x} \otimes F_{1}+\sigma_{x} \otimes \sigma_{x} \otimes F_{2}$

$-\sigma_{x} \otimes I \sigma_{y} \otimes F_{1}+\sigma_{x} \otimes \sigma_{x} \otimes F_{2}$

$-\sigma_{x} \otimes F_{1} \otimes F_{1}+I \sigma_{y} \otimes F_{2} \otimes F_{1}+\sigma_{x} \otimes \sigma_{x} \otimes F_{2}$

$-\sigma_{x} \otimes F_{1} \otimes F_{1}-I \sigma_{y} \otimes F_{2} \otimes F_{1}+\sigma_{x} \otimes \sigma_{x} \otimes F_{2}$

$I \sigma_{y} \otimes F_{1} \otimes F_{1}-\sigma_{x} \otimes F_{2} \otimes F_{1}+\sigma_{x} \otimes \sigma_{x} \otimes F_{2}$

$-I \sigma_{y} \otimes F_{1} \otimes F_{1}-\sigma_{x} \otimes F_{2} \otimes F_{1}+\sigma_{x} \otimes \sigma_{x} \otimes F_{2}$

$-\sigma_{x} \otimes \sigma_{x} \otimes F_{1}+\sigma_{x} \otimes \sigma_{x} \otimes F_{2}$ 
